\title{
Rice diversity panel provides accurate genomic predictions for complex traits in the progenies of biparental crosses involving members of the panel
}

\author{
M. Ben Hassen ${ }^{1}$ - T. V. Cao ${ }^{2}$. J. Bartholomé ${ }^{2} \cdot$ G. Orasen ${ }^{1} \cdot$ C. Colombi ${ }^{3} \cdot$ J. Rakotomalala $^{4} \cdot$ L. Razafinimpiasa ${ }^{4}$. \\ C. Bertone ${ }^{1} \cdot$ C. Biselli ${ }^{5} \cdot$ A. Volante ${ }^{6} \cdot$ F. Desiderio $^{5} \cdot$ L. Jacquin $^{2} \cdot$ G. Valè $^{6} \cdot$ N. Ahmadi ${ }^{2}$ (D)
}

Received: 6 June 2017 / Accepted: 4 November 2017 / Published online: 14 November 2017

(c) The Author(s) 2017. This article is an open access publication

\begin{abstract}
Key message Rice breeding programs based on pedigree schemes can use a genomic model trained with data from their working collection to predict performances of progenies produced through rapid generation advancement. Abstract So far, most potential applications of genomic prediction in plant improvement have been explored using cross validation approaches. This is the first empirical study to evaluate the accuracy of genomic prediction of the performances of progenies in a typical rice breeding program. Using a cross validation approach, we first analyzed the effects of marker selection and statistical methods on the accuracy of prediction of three different heritability traits in a reference population (RP) of 284 inbred accessions. Next, we investigated the size and the degree of relatedness with the progeny population (PP) of sub-sets of the RP that maximize the accuracy of prediction of phenotype across generations, i.e., for $97 \mathrm{~F}_{5}-\mathrm{F}_{7}$ lines derived from biparental crosses between 31 accessions of the RP. The extent of linkage disequilibrium was high $\left(r^{2}=0.2\right.$ at $0.80 \mathrm{Mb}$ in $\mathrm{RP}$ and at $1.1 \mathrm{Mb}$ in PP). Consequently, average marker density above one per $22 \mathrm{~kb}$ did not improve the accuracy of predictions in the RP. The accuracy of progeny prediction varied greatly depending on the composition of the training set, the trait, LD and minor allele frequency. The highest accuracy achieved for each trait exceeded 0.50 and was only slightly below the accuracy achieved by cross validation in the RP. Our results thus show that relatively high accuracy $(0.41-0.54)$ can be achieved using only a rather small share of the RP, most related to the PP, as the training set. The practical implications of these results for rice breeding programs are discussed.
\end{abstract}

\section{Introduction}

Genomic selection (GS) arose from the conjunction of new high-throughput marker technologies and new statistical methods (Meuwissen et al. 2001). GS allows analysis of the genetic architecture for complex traits in the framework of

Communicated by Marcos Malosetti.

Electronic supplementary material The online version of this article (https://doi.org/10.1007/s00122-017-3011-4) contains supplementary material, which is available to authorized users.

N. Ahmadi

nourollah.ahmadi@cirad.fr

1 Department of Agriculture and Environmental Sciences, University of Milan, Via Giovanni Celoria, 2, 20133 Milan, Italy

2 Cirad, UMR AGAP, Avenue Agropolis, 34398 Montpellier Cedex 5, France

3 Fondazione Parco Tecnologico Padano, Via Einstein, Loc. Cascina Codazza, 26900 Lodi, Italy infinitesimal model effects. It consists in (1) using all markers (often large numbers) simultaneously to build a model

4 FOFIFA, Antananarivo, Madagascar

5 CREA-Council for Agricultural Research and Economics, Research Center for Genomics and Bioinformatics, Via S. Protaso 302, 29017 Fiorenzuola d'Arda, PC, Italy

6 CREA-Council for Agricultural Research and Economics, Research Center for Cereal and Industrial Crops, S. S. 11 to Torino Km 2.5, 13100 Vercelli, Italy 
of genotype-phenotype relationships in a training population (TP), thus accounting also for linkage disequilibrium (LD) among markers, and (2) using the model to predict the genomic estimate of breeding values (GEBV) of candidates in a breeding population (CP) (Meuwissen et al. 2001; Jannink et al. 2010). The effectiveness of GS depends, among other factors, on the degree of correlation between the predicted GEBV and the true genetic value, i.e., the accuracy of prediction. In practice, the accuracy of prediction is evaluated by the correlation between GEBV and the realized phenotype.

Prospects for the applications of GS in plant breeding have given rise to many studies using simulations or experimental data. The effect of the statistical method on the accuracy of GEBV has been widely analyzed (Heslot et al. 2012). The general conclusion is that there is no single best statistical method and that the accuracy of the different methods depends on other factors, such as the characteristics of the target trait, the density and distribution of the markers, the size and the structure of the TP, and the degree of relatedness between TP and CP. The characteristics of the target trait reported to influence the accuracy of predictions include heritability, the number of QTLs, the distribution of their allelic effects and frequencies, and the relative magnitude of additive and non-additive genetic variance (Hayes et al. 2009a; Jannink et al. 2010; Howard et al. 2014; Burstin et al. 2015). Regarding marker density, empirical studies have confirmed the theoretical stance that marker density should be high enough to ensure strong linkage disequilibrium (LD) with at least one marker for each QTL (Lorenzana and Bernardo 2009; Lorenz et al. 2011; Heffner et al. 2011; Poland et al. 2012; Heslot et al. 2013). Another set of factors that strongly influence the accuracy of predictions includes the size of the TP, its structure, and its relatedness with the CP. Accounting for population structure through stratified sampling in the TP can significantly improve the accuracy of the predictions (Albrecht et al. 2011; Grenier et al. 2015; Isidro et al. 2015). Methods have been developed to optimize the composition of the TP (Rincent et al. 2012; Akdemir et al. 2015), by maximizing the expected reliabilities for a given set of individuals.

GS issues that need more thorough empirical studies include the evaluation of accuracy of genomic prediction for making selection decisions in pedigree breeding within the progeny of biparental crosses (Desta and Ortiz 2014). Beyene et al. (2015) compared the genetic gain for grain yield realized in eight biparental crosses of maize under GS associated with rapid cycling, with conventional pedigree breeding and reported that the average genetic gain per year under GS was three times higher than that achieved by conventional breeding. However, such a GS breeding approach required separate model training for each biparental cross and phenotyping of the first generation of progeny, which increase the costs and duration of breeding cycles. A second approach, recently investigated in a number of crops, is the use of a reference set to train the prediction model and the use of this model to predict the performance of progenies from biparental crosses between members of the panel. For instance, Hofheinz et al. (2012) used a reference set of 310 inbred sugar beet lines to predict the test cross value of 56 inbred progeny derived from eight crosses between six lines of the reference set, and reported average prediction accuracy of 0.79 for sugar content. Sallam et al. (2015) used a training set of 168 barley lines and five sets of 96 progeny lines, representative of the breeding lines developed in five consecutive years (the training set included the parents of the progeny sets) and reported a prediction accuracy of around 0.50 for grain yield. Likewise, Gezan et al. (2017) used a panel representative of the Florida University strawberry breeding program and sets of progenies derived from the circular mating of 31 members of the panel and reported a prediction accuracy ranging from 0.16 to 0.77 depending on the traits and model fitting method used. However, this approach faces the challenge of differences in LD and allele frequencies between the reference panel and the progenies of individual biparental crosses, requiring thorough management of marker density. It also raises the question of the choice of prediction model to capture either markers' LD with QTLs or the marker-based relationship between the training set and the progenies sets, both of which require knowledge of the genetic architecture of the target traits (Zhong et al. 2009; Zhang et al. 2017).

Rice (Oryza sativa) is the world's most important staple food and will continue to be so in the coming decades. Genetic improvement is one of the major pillars of sustainable adaptation of rice production to ongoing global changes (Atlin et al. 2017). GS is expected to accelerate genetic gain for traits such as yield potential and adaptation to constraints related to climate change and the efficient use of resources (water, nitrogen, etc.) (Ashikari 2017; Atlin et al. 2017). So far, however, GS studies on rice have mainly explored the cross validation approach within diversity panels (Table 1 ).

Here we report the first empirical study that assesses the accuracy of genomic prediction among the progeny of rice biparental crosses using a reference panel for model training. It was undertaken in the framework of a rice breeding program conducted according to the most common rice breeding scheme, i.e., pedigree breeding within the progenies of biparental crosses, the parents being chosen within a working collection of inbred accessions. The reference panel was composed of 284 inbred accessions of the breeding program's working collection. The progeny population was composed of 97 inbred lines derived from 36 biparental crosses between 31 accessions of the working collection. The main objectives of this study were to investigate (1) the size and the degree of relatedness of the reference panel that 


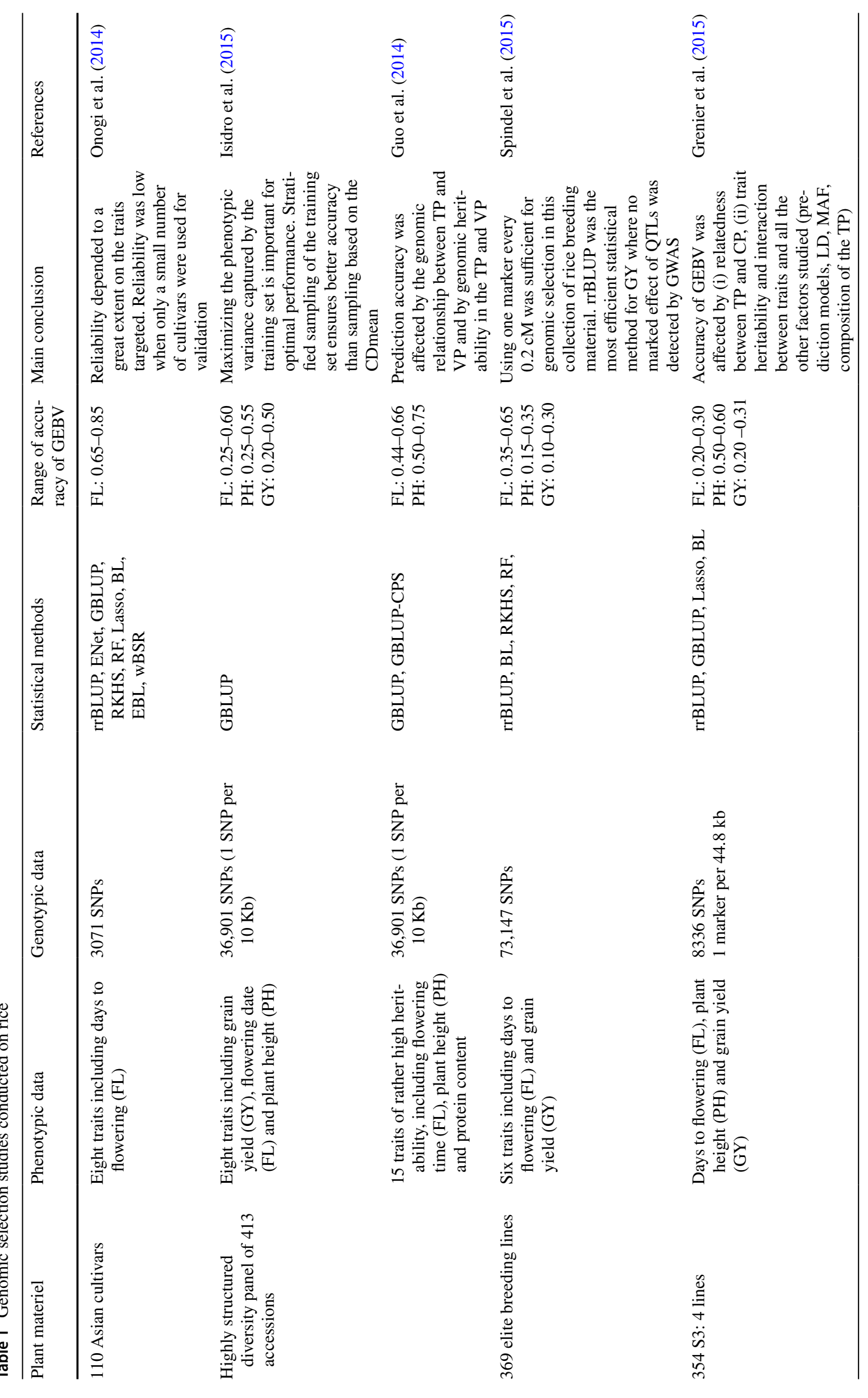




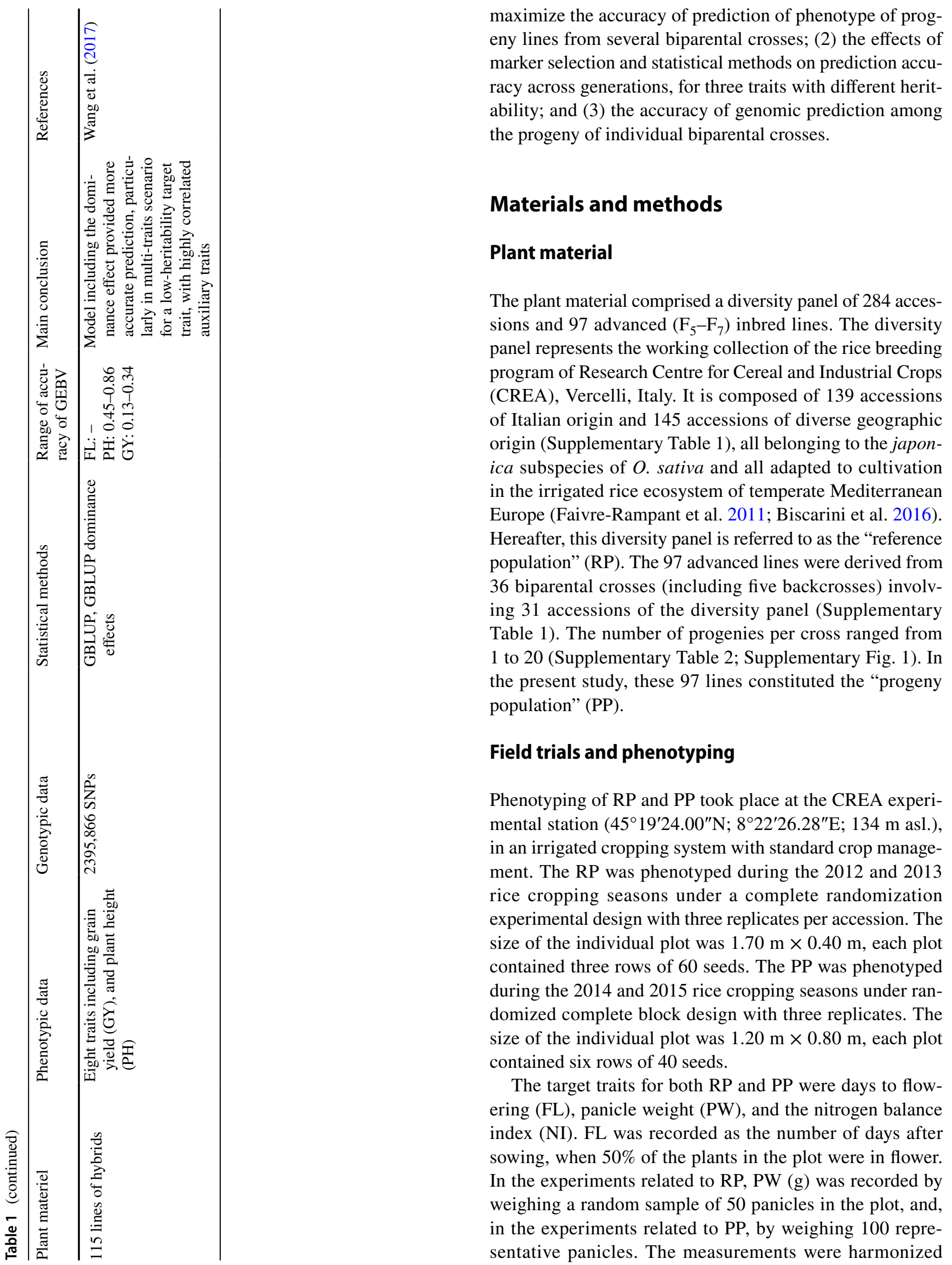


to represent the weight of 100 panicles. NI, an indicator of the plant nitrogen status (Tremblay et al. 2012), was recorded using a Dualex ${ }^{\mathrm{TM}}$ instrument (Goulas et al. 2004), 7 to 10 days after the flowering date, a period during which the nitrogen status of the plant is stable. In each plot, three measurements were made on the adaxial and the abaxial sides of a flag leaf on three plants. The 18 measurements were then averaged to obtain a plot level NI.

\section{Analysis of phenotypic data}

Phenotypic data for each trait and each population were analyzed separately using the proc mixed procedure of SAS 9.2 (SAS Institute, Cary NC, USA). The mixed model used for the RP was:

$Y_{i j k}=\mu+g_{i}+y_{j}+(g y)_{i j}+e_{i j k} \quad$ (RP model),

where $Y_{i j k}$ is the observed phenotype of genotype $i$ in year $j$ and in plot $k, \mu$, the overall mean, $g_{i}$, the genotype effect, $y_{j}$, the year effect, $(g y)_{i j}$, the interaction between genotype $i$ and year $j$, and $e_{i j k}$ the residual. Except for the overall mean, all the effects were considered random and the variance components were tested using the Wald Z-statistic tests, which are appropriate for large sample sizes.

The mixed model used for the PP was:

$Y_{i j k}=\mu+g_{i}+y_{j}+r(y)_{j k}+(g y)_{i j}+e_{i j k} \quad$ (PP model),

where $Y_{i j k}, \mu, g_{i}, y_{j},(g y)_{i j}$ and $e_{i j k}$ have the same meaning as in the RP model, and $r(y)_{j k}$, is the replicate within year effect. As in the previous model, all the effects except $\mu$ were considered random.

A model-based diagnostic analysis was run for each field trial and each trait within the mixed model framework above, to detect potential outliers among the individual data points (plot level). The restricted likelihood distance (RLD) output of the diagnostic procedure was used to identify outliers. RLD is a global measure of the influence of the observations jointly on all parameters. If $\varphi$ denotes the collection of all parameters in the model, i.e., including fixed $(\beta)$ and random $(\theta)$, then $R L D(U)=2\{l R(\hat{\varphi})-l R(\hat{\varphi}(U))\}$ is twice the difference between the restricted log-likelihood evaluated at the full-data estimates $\hat{\varphi}$ and at the reduced-data estimates $\hat{\varphi}(\mathrm{U})$. The distribution of the RLD values of the accessions was inspected visually and when one was considered too high, the corresponding plot data were compared, and outlier plots were either corrected or discarded. This procedure resulted in the elimination of five data points (of PW) in the 2013 field trial involving the RP. The eliminated data were considered as missing in the following steps of data analysis.

In addition to the above-mentioned diagnostic analysis, spatial homogeneity of the experimental fields, where RP phenotyping took place under a complete randomization design, was surveyed by visual analysis of the heat-map of the residuals (Supplementary Fig. 2). A slight discrepancy in the random distribution of the residuals was observed for NI only.

Broad sense heritability of accession means, $H^{2}$, was calculated for each trait in each population using the formula of Holland et al. (2003) as follows:

$$
H^{2}=\frac{\sigma_{g}^{2}}{\sigma_{g}^{2}+\frac{\sigma_{g y}^{2}}{n y}+\frac{\sigma_{e}^{2}}{n r}}
$$

where $n y$ represents the mean number of years in which the accessions were tested and $n r$, the mean number of plots per accession across years. The means were calculated as harmonic means. Finally, adjusted means of accessions $\left(\hat{Y}_{i}=\hat{\mu}+\hat{g}_{i}\right.$, with $g$, as random effect) were extracted for each trait to be used as phenotypes in the genomic prediction models.

\section{Genotyping and genotypic data}

The genotyping procedure is detailed in Biscarini et al. (2016). Briefly, genomic DNA was isolated from 3-weekold leaves using the DNeasy Plant Mini Kit (QIAGEN, Milan, Italy) with a TECAN Freedom EVO150 liquid handling robot (TECAN Group Ltd, Männedorf, Switzerland). DNA digestion was performed using ApeKI restriction enzyme. Digested DNAs were ligated to 12 of 0.6/adapter pairs (optimized to guarantee good quality libraries in rice), and the 96-plex library constructed according to the genotyping by sequencing (GBS) protocol. The libraries were loaded into a Genome Analyzer II (Illumina, Inc., San Diego, USA) for sequencing. The Tassel GBS pipeline v3.0 (Glaubitz et al. 2014) was used to filter the raw data, sequence alignment to the rice reference genome (Os-Nipponbare-Reference-IRGSP-1.0), and for SNP calling. The procedure yielded 246,554 SNPs with a call rate $\geq 80 \%$. Filtering of the matrix for missing data with a threshold of $20 \%$ led to 70,530 SNPs with an average rate of $9.2 \%$ missing data. Missing SNP genotypes were then imputed using the FILLIN (Fast, Inbred Line Library ImputatioN) algorithm in the Tassel GBS pipeline v3.0, with default settings. Filtering of this matrix for the rate of heterozygoty (threshold of 5\%) and for a minor allele frequency (MAF, threshold of 2.5\%) among the RP accessions and PP lines, considered together, led to a final working set of 43,686 SNP loci. The genotypic data are available at http://tropgenedb.cirad.fr/tropgene/ JSP/interface.jsp?module=RICE, (Choose Tab Studies) as GS-Ruse_CREA_GBSgenotype_RP\&PP. 


\section{Genotypic characterization of RP and PP}

The genetic structure of the two populations was analyzed jointly using a distance-based method. First, a matrix of 4824 SNPs was extracted from the working genotypic dataset of 43,686 SNPs, by discarding loci that had imputed data and by imposing a minimum distance of $10 \mathrm{~kb}$ between two adjacent loci. Then an unweighted neighbor-joining tree based on a simple matching matrix was constructed using DarWin v6 (Perrier and Jacquemoud-Collet 2006).

Pairwise LD between SNP loci was calculated separately in RP and PP at the level of the individual chromosome, using the working genotypic dataset of 43,686 SNPs and the $r^{2}$ estimator proposed by Rogers and Huff (2009) for non-phased genotypic data.

\section{Genomic prediction methods}

Three statistical methods were tested: genomic best linear unbiased prediction (GBLUP), reproducing kernel Hilbert spaces regressions (RKHS) and BayesB (Meuwissen et al. 2001). The GBLUP method (VanRaden 2008) was implemented using the Expectation-Maximization convergence algorithm and the genomic matrix $G=X X^{\prime}, X$ being the centered genotype matrix containing values of $-1,0$ and 1 , with $N \times P$ dimension, where $N$ is the number of entries and $P$ the number of markers. For the RKHS regression (Gianola and van Kaam 2008), the Gaussian kernel $K\left(x_{i}, x_{j}\right)=\exp \left(-h|| x_{i}-x_{j} \|^{2}\right)$ was used to build the kernel matrix (or the Gram matrix) between the marker genotype vectors $x_{i}$ and $x_{j}$, where $(i, j) \in\{1, \ldots, N\}^{2}$. The rate of decay parameter $h$, also known as the bandwidth parameter, was estimated using the $k$-folds cross validation method implemented in the Tune_kernel_Ridge_MM function of the $\mathrm{R}$ package KRMM. The R package kernlab (Karatzoglou et al. 2004) was used to compute the kernel matrix. Both GBLUP and RKHS methods were implemented using the KRMM package (https://cran.r-project.org/web/packages/KRMM/ index.html) described by Jacquin et al. (2016). For BayesB, the model that specified two component mixtures prior with a point of mass at zero and a scaled- $t$ slab for marker effect (Meuwissen et al. 2001) was implemented using the BGLR statistical package (Pérez and de los Campos 2014). The default parameters for prior specification were used and the number of iterations for the Markov chain Monte Carlo (MCMC) algorithm was set to 12,000 with a burn-in period of 2000 .

\section{Construction of the incidence matrices}

Twenty-one incidence matrices were constructed to investigate the effect of LD (7 threshold levels) and MAF (3 threshold levels), on the accuracy of genomic predictions within the RP. The seven LD thresholds, $r^{2} \leq 0.25, \leq 0.3$ $6, \leq 0.49, \leq 0.64, \leq 0.81, \leq 0.98$ and $\leq 1$, were chosen so as to correspond to the square of seven thresholds of Pearson correlation between genotype at each pair of loci of a given chromosome $(-0.5 \leq r \leq+0.5,-0.6 \leq r \leq+0.6$, $-0.7 \leq r \leq+0.7,-0.8 \leq r \leq+0.8,-0.9 \leq r \leq+0.9$, $-0.99 \leq r \leq+0.99,-1 \leq r \leq+1)$. The choice of the three MAF thresholds ( $\geq 5, \geq 10$, and $\geq 20 \%$ ) was intended to represent its distribution (first quartile, median and third quartile) within RP and PP.

The incidence matrices were constructed as follows: (1) using the genotypic dataset $(N=289$ entries and $P=43,686$ SNPs), markers were selected based on the three MAF thresholds ( $\geq 5, \geq 10$, and $\geq 20 \%$ ); (2) for each of the three resulting matrices, the pairwise LD between markers was calculated for each chromosome; (3) for each marker and for each LD threshold, redundancy information was computed as the number of times the pairwise LD with other markers was above the LD threshold; (4) markers with a redundancy level above an empirical threshold of 30 were discarded. This empirical threshold of redundancy represented a good compromise between our two objectives, one to reduce redundancy, which varied widely between SNPs, the other to dispose of an ample range of marker density among the 21 incidence matrices. As a result, marker density ranged from 8.7 to $83.5 \mathrm{SNP}$ per Mb for the seven LD thresholds under $\mathrm{MAF} \geq 5 \%$, from 5.0 to 69.9 under MAF $\geq 10 \%$ and from 3.1 to 52.4 under MAF $\geq 20 \%$ (Table 2).

\section{Cross validation experiments}

The cross validation experiments used $189(2 / 3)$ of the 284 accessions of the RP as the training set and the remaining 95 $(1 / 3)$ accessions as the validation set. Each cross validation experiment was repeated 100 times using 100 independent partitioning of the accessions into the training set and validation set. For each independent partitioning, the correlation between the predicted and the observed phenotype was calculated, so as to obtain 100 correlations for each cross validation experiment. The accuracy of each cross validation experiment was computed as the mean value of the 100 correlations.

A total of 189 cross validation experiments were undertaken combining the above-described seven LD threshold levels, three MAF threshold levels, three prediction methods, and the three phenotypic traits. The same 100 independent partitioning of the training and validation sets was used for all 189 cross validation experiments.

\section{Genomic prediction across generations}

Six scenarios, representing different degrees of relatedness between the training set and the progeny set and different 
Table 2 Size of the incidence matrices used in the cross validation experiments in the reference population

\begin{tabular}{|c|c|c|c|c|c|c|}
\hline \multirow[t]{3}{*}{$\mathrm{LD}\left(r^{2}\right)$} & \multicolumn{6}{|c|}{ Minor allele frequency (MAF) } \\
\hline & \multicolumn{2}{|l|}{$\geq 5 \%$} & \multicolumn{2}{|l|}{$\geq 10 \%$} & \multicolumn{2}{|l|}{$\geq 20 \%$} \\
\hline & $N$ & $D$ & $N$ & $D$ & $N$ & $D$ \\
\hline$\leq 0.25$ & 3322 & 8.7 & 1927 & 5.0 & 1173 & 3.1 \\
\hline$\leq 0.36$ & 5365 & 14.0 & 3450 & 9.0 & 2270 & 5.9 \\
\hline$\leq 0.49$ & 8324 & 21.7 & 5738 & 14.9 & 4013 & 10.5 \\
\hline$\leq 0.64$ & 12,099 & 31.5 & 8744 & 22.8 & 6095 & 15.9 \\
\hline$\leq 0.81$ & 16,923 & 44.1 & 12,652 & 34.2 & 8917 & 23.2 \\
\hline$\leq 0.98$ & 28,164 & 73.3 & 23,119 & 60.2 & 16,750 & 43.6 \\
\hline$\leq 1$ & 32,066 & 83.5 & 26,845 & 69.9 & 20,104 & 52.4 \\
\hline
\end{tabular}

$N$ total number of SNPs, $D$ SNP density per Mb
Table 3 Scenarios for genomic prediction across generations

\begin{tabular}{lll}
\hline Scenario & Training set & Validation set \\
\hline S1 & 31 parents & 97 progeny \\
S2 & 58 related accessions & 97 progeny \\
S3 & 31 parents +58 related accessions & 97 progeny \\
S4 & 31 parents + 252 accessions & 97 progeny \\
S5 & 252 accessions, excluding the parents & 97 progeny \\
S6 & 100 random sampling of 31 accessions, & 97 progeny \\
& excluding the parents \\
\hline
\end{tabular}

sizes of the training set, were considered (Table 3). To this end, first, using pairwise Euclidian distances between each parental line and other accessions of the RP, the three closest accessions to each of the 31 parental accessions were identified. These accessions were then pooled to form the most related subset. Pooling led to a total of 58 accessions, because the closest accessions for some parents also happened to be the closest for other parents. Finally, this subset was combined, or not, with the parental lines and with the other accessions of RP to constitute the six training sets of the six prediction scenarios. For each scenario, the correlation between the predicted and the observed phenotypes of the 97 progeny lines was calculated, and represents the accuracy of the prediction experiment. In the case of scenario S6, in which the 31 accessions of the training set were randomly sampled 100 times from the RP excluding the parents, prediction accuracy was computed as the mean value of the 100 correlations between the predicted and the actual phenotypes of the 97 progeny lines. The objective of this random sampling was to reduce the risk of over-/under-estimation of prediction accuracy in this scenario. Comparisons between scenarios were consequently based on progeny prediction accuracy (PPA) data for the non-replicated prediction experiments, and on the average PPA for the replicated experiments in scenario S6.
The six scenarios were implemented with seven incidence matrices corresponding to the seven thresholds of LD used in the cross validation experiments, a unique MAF threshold of $\geq 5 \%$, and three prediction methods (GBLUP, RKHS and BayesB). The accuracy observed under scenarios S1, S2 and S3 was also compared with the accuracy obtained by three training sets of equivalent sizes selected using the dedicated CDmean optimization method (Rincent et al. 2012).

To explore the accuracy of progeny prediction within the progenies of individual crosses, the correlation between the predicted and the observed phenotypes of the progeny lines of two crosses represented by a reasonably high number of advanced lines (Eurosis $\times$ Handao- 11 and Giano $\times$ Vialone-Nano, represented by 20 lines and 9 lines, respectively) were also computed separately.

\section{Analysis of sources of variation in the accuracy of genomic prediction}

The accuracy data $(r)$ of all prediction experiments were transformed into a $Z$-statistic using the equation: $Z=0.5\{\ln [1+r]-\ln [1-r]\}$ and analyzed as a dependent variable in an analysis of variance. After estimation of confidence limits and means for $\mathrm{Z}$, these were transformed back to $r$ variable. For each trait, a separate ANOVA was performed for the correlations of all the PPA and of the average PPA in the progeny prediction experiments. In each case, ANOVA was performed to partition the variance of accuracy into different sources, with all effects declared as fixed, and following two models. The first model compared the effects of LD, MAF and prediction method in the cross validation experiments, and the effect of LD, scenario and prediction method in the progeny prediction experiments, with no interaction. The second model accounted for all the principal effects as well as for all possible first-order interactions. 


\section{Results}

\section{Phenotypic diversity of the three traits investigated}

The three traits investigated in the RP and PP populations exhibited a Gaussian distribution (Fig. 1). For all three traits, the extent of phenotypic diversity was broader in the RP than in the PP. Moreover, the distribution of NI and PW in the PP remained among the lowest values for these traits, leading to lower mean values. The narrower phenotypic diversity of PP is probably linked to its narrower genetic diversity (see below).

Separate ANOVA conducted in the RP and in the PP revealed a very highly significant effect of entry or genotype for the three traits (Table 4). The year effect was not significant, whereas the effects of genotype by year interaction were significant.

$H^{2}$ was rather high for FL or PW, with $H^{2}>0.8$ and moderate for NI $\left(H^{2}=0.56\right)$, in the RP. For the PP, $H^{2}$ was high for all traits $\left(H^{2} \geq 0.8\right)$. The precision of the $H^{2}$ estimates was reasonably high, as the standard errors ranged between 0.007 and 0.052 .

\section{Genotypic data and genetic diversity}

The 43,686 SNP markers were unevenly distributed along the chromosomes. While the average marker density was 1 SNP per $8.8 \mathrm{~kb}$, it ranged from one SNP every $5.1 \mathrm{~kb}$ on chromosome 11 to one SNP every $12.6 \mathrm{~kb}$ on chromosome 3 (Supplementary Table 3; Supplementary Fig. 3). The distance between a pair of adjacent SNPs ranged from 0.001 to $644 \mathrm{~kb}$, with a median of $1.20 \mathrm{~kb}$. The distance was $<20 \mathrm{~kb}$ in almost $90 \%$ of the pairs of adjacent markers and $<100 \mathrm{~kb}$ in $98.8 \%$. The distance was $>100 \mathrm{~kb}$ in 500 pairs of adjacent SNPs, $>200 \mathrm{~kb}$ in 114 pairs and $>500 \mathrm{~kb}$ in only one pair.

Even though the markers whose MAF was below 2.5\% had been discarded, the distribution of MAF was still skewed toward low frequencies. The proportion of loci with a MAF $<10 \%$ was $38.5 \%$ in the RP and $30 \%$ in the PP. The differences in allele frequency between the two populations were mainly quantitative, not qualitative: for $93 \%$ of loci, the minor allele in RP was also the minor allele in PP, and the average MAF for these loci was 24.6 in RP and 22.7 in PP. Conversely, for $7 \%$ of loci, the minor allele in RP (average frequency of $42.7 \%$ ) become a major allele in PP with an average frequency of $56.4 \%$, suggesting the accentuation of allele frequency disequilibrium observed in RP. This

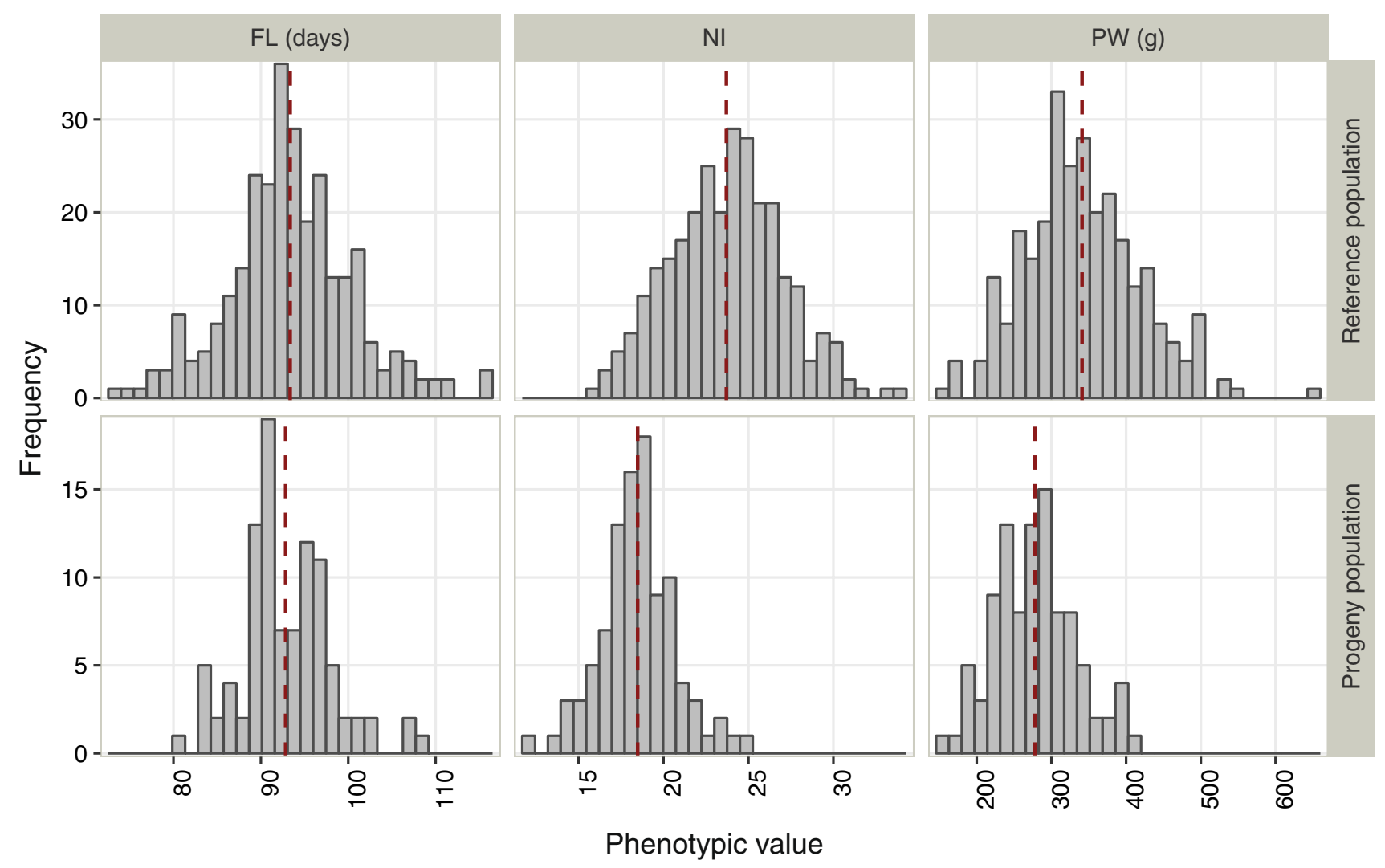

Fig. 1 Distribution of phenotypic values for days to flowering (FL), nitrogen balance index (NI) and panicles weight (PW) in the reference and the progeny populations 
Table 4 Variance components of three phenotypic traits in the reference and progeny populations

\begin{tabular}{lllcc}
\hline Population & Factors & FL & NI & PW \\
\hline Reference population & Genotype & $47.78^{* * *}$ & $6.17 * * *$ & $5023.13 * * *$ \\
& Year & $16.82 \mathrm{NS}$ & $2.96 \mathrm{NS}$ & $222.18 \mathrm{NS}$ \\
& Year $\times$ genotype & $4.36 * * *$ & $4.08 * * *$ & $889.8^{* * *}$ \\
& Residual & 5.95 & 16.74 & 2378.04 \\
Progeny population & $H^{2}(\mathrm{SE})$ & $0.937(0.007)$ & $0.558(0.052)$ & $0.852(0.018)$ \\
& Genotype & $23.2^{* * *}$ & $4.12^{* * *}$ & $2698.61^{* * *}$ \\
& Year & $55.47 \mathrm{NS}$ & $4.99 \mathrm{NS}$ & $16.19 \mathrm{NS}$ \\
& Year $\times$ genotype & $7.38^{* * *}$ & $0.7 * * *$ & $415.03 * * *$ \\
& Residual & 2.27 & 3.72 & 554.11 \\
& $H^{2}(\mathrm{SE})$ & $0.849(0.031)$ & $0.798(0.041)$ & $0.899(0.021)$ \\
\hline
\end{tabular}

$F L$ days to flowering, NI nitrogen balance index, $P W 100$ panicle weight, $H^{2}$ broad sense heritability, $N S$ not significant

$* * *$ Significant at $p=0.001$ tendency was confirmed by the allele frequencies in loci with the lowest MAF $(<10 \%)$ in RP. In these loci, which represented $16 \%$ of the total, only one minor allele in RP became a major allele in PP, while 951 minor alleles in RP were lost in PP and the corresponding loci became monomorphic with the major allele of RP. The MAF differences between RP and PP were even more pronounced for the smallest incidence matrix of 3322 SNPs, with a MAF $<5 \%$ for $25.7 \%$ of the loci and $9.2 \%$ of monomorphic loci.

The decay of LD along physical distance is presented in Fig. 2 and Supplementary Table 4. For between-marker distances of 0 to $25 \mathrm{~kb}$, the $r^{2}$ value reached 0.62 and 0.66 in the RP and in the PP, respectively. In the RP, the $r^{2}$ value dropped to half its initial level at around $350 \mathrm{~kb}$ and reached 0.2 at $800 \mathrm{~kb}$ and 0.1 at $2.9 \mathrm{Mb}$. As expected, the decay of LD was slower in the PP, reaching an $r^{2}$ of 0.2 at $1.1 \mathrm{Mb}$ and 0.1 at 3.9 Mb. Some differences in the speed of LD decay were observed between chromosomes, with the highest speed in chromosome $11\left(r^{2}=0.21\right)$ reached between 200 and $225 \mathrm{~kb}$ in the RP and an $r^{2}$ of 0.20 reached between 300 and $350 \mathrm{~kb}$, and the lowest in chromosome 5, with an $r^{2}$ of 0.2 at $1-1.5 \mathrm{Mb}$ in both populations.

The genetic diversity analysis of the RP led to two major clusters corresponding to the well-known temperate japonica (217 accessions) and tropical japonica (67 accessions) sub-groups (Fig. 3). The majority of the temperate japonica accessions are of European origin. The majority of tropical japonica accessions originate from the American continent. Interestingly, the average values for the three phenotypic traits investigated differed significantly in the two groups: 92 and 98 days for FL, 24.5 and 21.8 for NI and 354 and $305 \mathrm{~g}$ for PW in the temperate and the tropical japonica groups, respectively. Among the 31 accessions involved in biparental crosses for the development of the PP lines, 24 belonged to the temperate japonica group and seven to the tropical japonica group. Including the PP in the diversity
Fig. 2 Patterns of decay in linkage disequilibrium in the reference population (red) and in the progeny population (gray). The curve represents the average $r^{2}$ among the 12 chromosomes and the bars represent the associated standard deviation (color figure online)

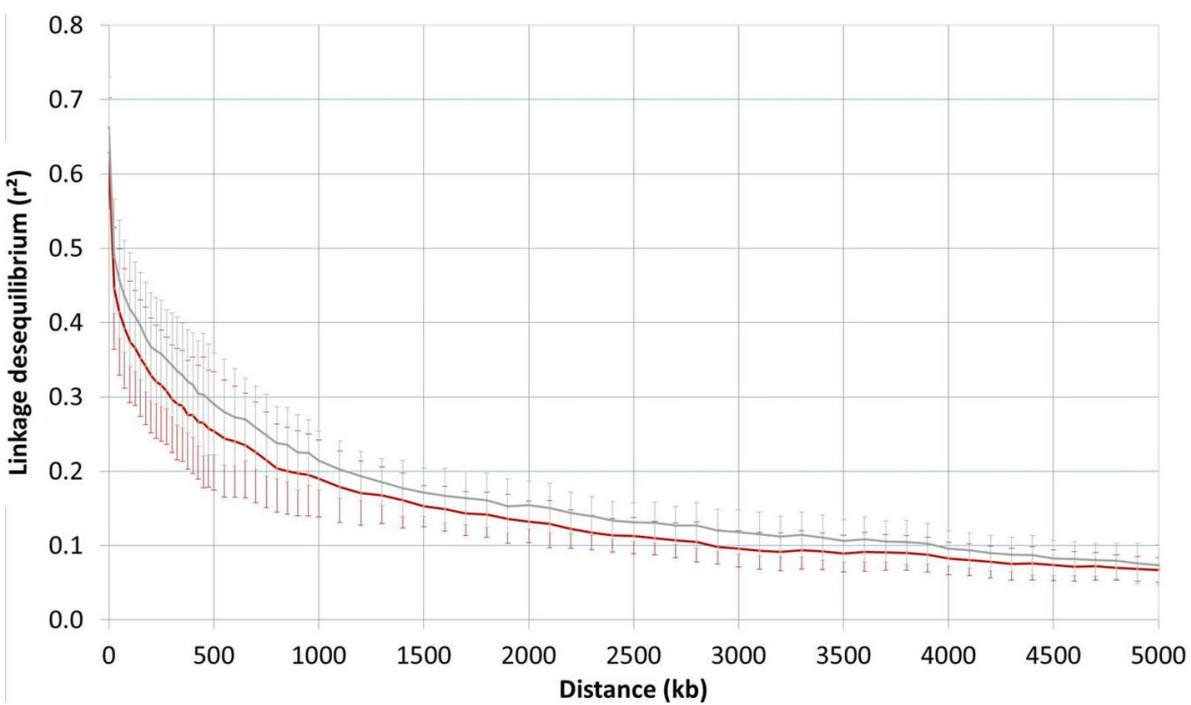




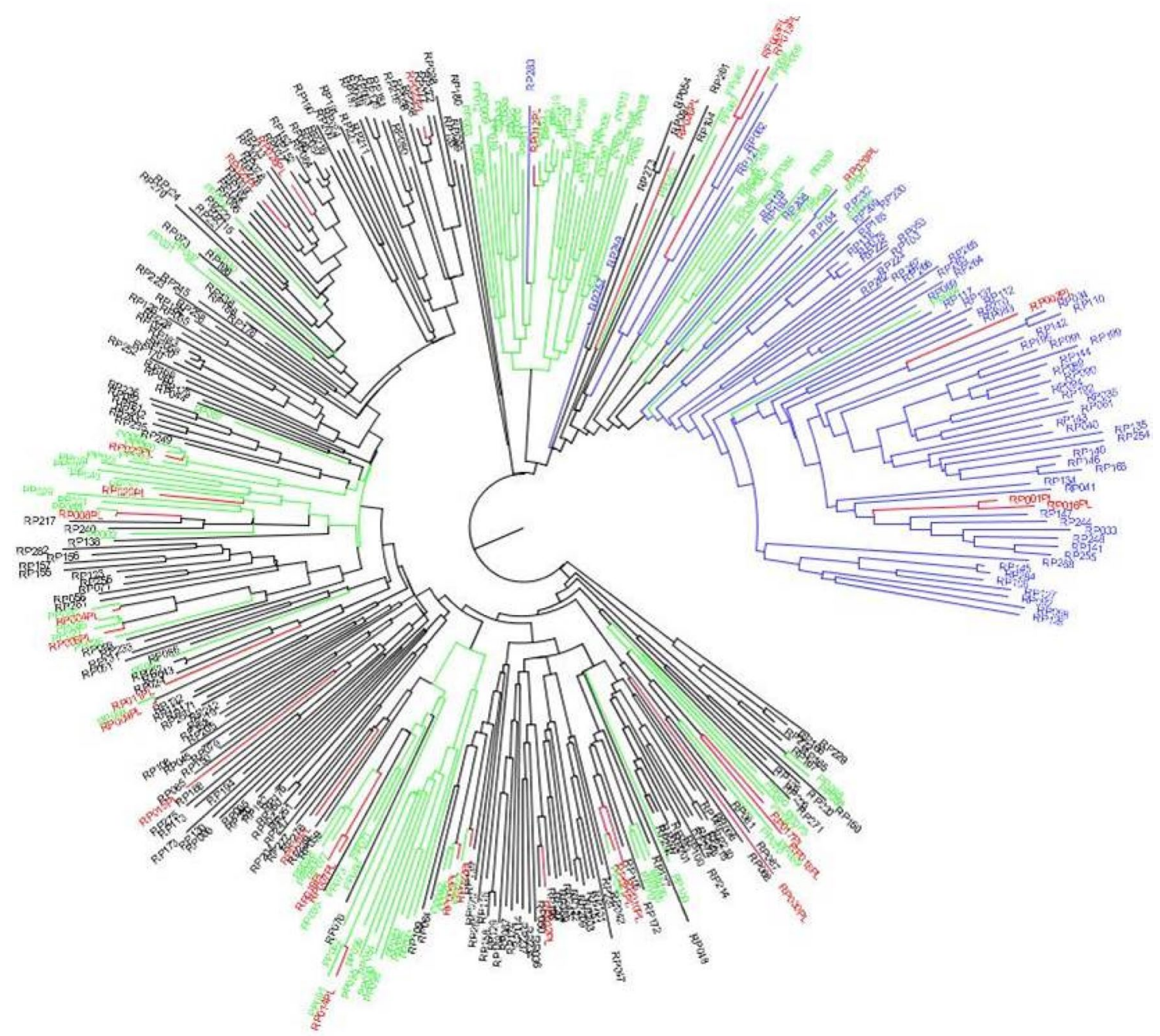

Fig. 3 Unweighted neighbor-joining tree based on simple matching distances constructed from the genotype of 284 accessions of the reference population (RP) and 97 lines of the progeny population (PP),

analysis did not modify the clustering into two groups, but only six progeny lines clustered with the tropical japonica group, while out of the 97 lines, a total of 43 derived from 11 crosses involving a tropical japonica donor. The remaining 37 PP lines derived from crosses involving a tropical japonica donor clustered with the temperate japonica group (Fig. 3; Supplementary Table 2).

\section{Accuracy of genomic prediction in the diversity reference panel}

The 189 cross validation experiments involving seven levels of LD, three levels of MAF, three prediction methods and the three phenotypic traits yielded average prediction accuracies (APA) ranging from 0.42 to 0.65 (Fig. 4; Supplementary Table 5). The overall APA for the FL trait (the average prediction accuracy over $7 \mathrm{LD}$ levels $\times 3 \mathrm{MAF}$ using 4824 SNP markers. Red: parental lines (PL); Black and blue: $\mathrm{RP}$ accessions belonging to tropical japonica and temperate japonica, respectively; Green: PP (color figure online)

levels $\times 3$ prediction methods $=63$ cross validation experiments) was 0.63 . The overall APA was 0.50 for NI and 0.59 for PW. Given the notable difference between traits, a model per trait was fitted to assess the effects of LD, MAF and statistical method. LD had significant effects on the APA of each trait. The MAF and method effects were significant only for FL and NI (Table 5). The LD threshold leading to the highest APA (0.60), considering the three traits, was $r^{2} \leq 0.64$ and $r^{2} \leq 0.81$. The LD threshold leading to the lowest APA (0.53 and 0.55) among the three traits was $r^{2} \leq 0.25$ and $r^{2} \leq 0.36$. NI was the trait most affected by variations in the LD threshold, with a gain in APA of $0.12(21.6 \%)$ between LD levels $\left(r^{2}=0.25\right.$ and $\left.r^{2}=0.64\right)$ giving the lowest, and the highest APA, respectively. The MAF threshold leading to the overall highest APA (0.58) was MAF $\geq 5 \%$. The higher MAF thresholds tested led to the same lower APA (0.57). Finally, the 


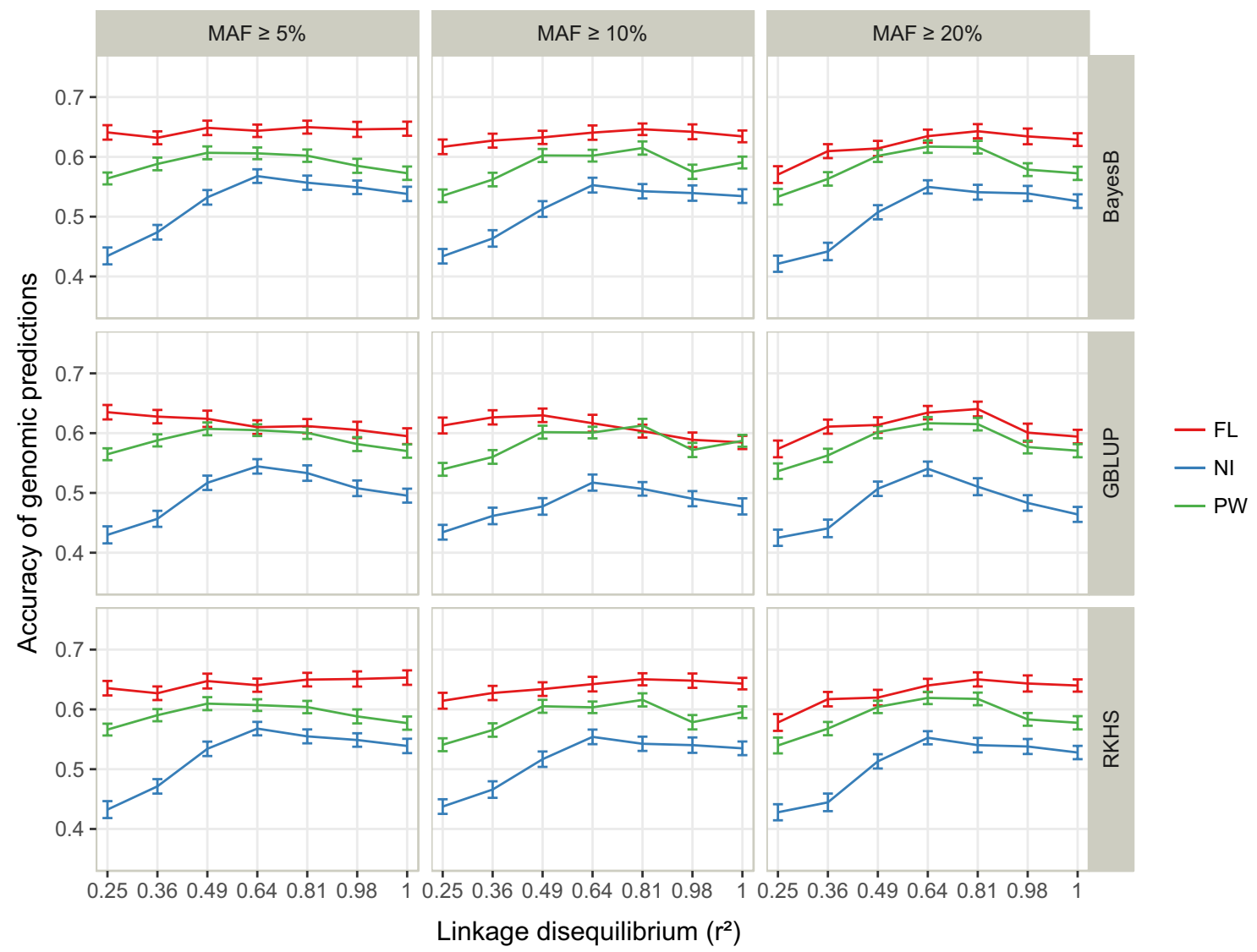

Fig. 4 Accuracy of genomic prediction in cross validation experiments in the reference population for days to flowering (FL), nitrogen balance index (NI) and 100 panicle weight (PW), obtained with 3 statistical methods, BayesB, GBLUP and RKHS

performances of the BayesB and RKHS methods were the same (0.58) and that of GBLUP was 0.56 .

All first rank interactions between the three factors affecting APA were significant except MAF $\times$ Method for NI and PW traits (Table 5). Among the three prediction methods, GBLUP was the most affected by the level of LD (Supplementary Fig. 4A). Indeed, for the LD threshold higher than 0.64, RKHS and BayesB performed significantly better than GBLUP with an increase in accuracy of up to 0.04 . In particular, this was the case of the FL and NI traits. The MAF $\times$ LD interaction led to diverging accuracies between MAF thresholds under the most stringent LD thresholds of $r^{2} \leq 0.49$ (Supplementary Fig. 4B).

Given these results, we decided to consider only one MAF threshold $(\geq 5 \%)$ in the following steps of the study (progeny prediction) and to focus on the analysis of the effect of LD and prediction method.

\section{Accuracy of genomic prediction across generations}

The 360 non-replicated experiments of genomic prediction of the progenies' phenotype, involving the first five scenarios $(\mathrm{S} 1-\mathrm{S} 5)$ of the relationship between the training set and the progeny set, seven LD thresholds, and three prediction methods led to progeny prediction accuracies (PPA) ranging from 0.23 to 0.51 (mean PPA 0.35 ) for the FL trait, 0.09 to 0.52 (mean PPA 0.33 ) for NI and 0.17 to 0.54 (mean PPA 0.38) for PW. The 72 replicated prediction experiments in scenario S6 led to an average PPA ranging from 0.05 to 0.22 (mean PPA 0.15) for the FL trait, 0.12 to 0.26 (mean PPA 0.21) for NI and 0.21 to 0.36 (mean PPA 0.30 ) for PW. The following comparisons of factors affecting PPA, especially the scenario factor, are based on PPA data for the non-replicated prediction experiments and on the average PPA for the replicated experiments of S6 (Fig. 5; Supplementary Table 6).

Variation in the scenario and in the LD factors significantly affected PPA for all traits. The effect of the prediction method was not significant (Table 6). The effects of interactions between scenario and LD and scenario and method were significant for the three traits. The interaction between LD and method was significant only for the FL trait. These interactions limit the interpretation of the individual main effects. Nevertheless, it is noteworthy that of the three factors, the scenario effect showed the greatest variances for the three phenotypic traits under the two ANOVA models, and, as expected, the trend of variations in PPA was related 
Table 5 ANOVA of factors affecting the transformed accuracy $(\mathrm{Z})$ of the 63 cross validation experiments per trait in the reference population

\begin{tabular}{|c|c|c|c|c|c|c|c|c|c|c|c|}
\hline Model & Trait & $R^{2}$ & $\mathrm{CV}$ & RMSE & Mean & Source & $d f$ & SS & MS & $F$ value & Prob $F$ \\
\hline \multirow[t]{18}{*}{ Main effects only } & \multirow[t]{6}{*}{ FL } & \multirow[t]{6}{*}{0.5650} & \multirow[t]{6}{*}{3.27} & \multirow[t]{6}{*}{0.024} & \multirow[t]{6}{*}{0.74} & Model & 10 & 0.0393 & 0.0039 & 6.75 & $<.0001$ \\
\hline & & & & & & Error & 52 & 0.0302 & 0.0006 & & \\
\hline & & & & & & Corrected total & 62 & 0.0695 & & & \\
\hline & & & & & & LD & 6 & 0.0127 & 0.0021 & 3.63 & 0.0044 \\
\hline & & & & & & MAF & 2 & 0.0068 & 0.0034 & 5.85 & 0.0051 \\
\hline & & & & & & Method & 2 & 0.0198 & 0.0099 & 17.02 & $<.0001$ \\
\hline & \multirow[t]{6}{*}{ NI } & \multirow[t]{6}{*}{0.9404} & \multirow[t]{6}{*}{2.82} & \multirow[t]{6}{*}{0.016} & \multirow[t]{6}{*}{0.56} & Model & 10 & 0.2014 & 0.0201 & 82.02 & $<.0001$ \\
\hline & & & & & & Error & 52 & 0.0128 & 0.0002 & & \\
\hline & & & & & & Corrected total & 62 & 0.2142 & & & \\
\hline & & & & & & $\mathrm{LD}$ & 6 & 0.1780 & 0.0297 & 120.8 & $<.0001$ \\
\hline & & & & & & MAF & 2 & 0.0055 & 0.0028 & 11.22 & $<.0001$ \\
\hline & & & & & & Method & 2 & 0.0179 & 0.0090 & 36.47 & $<.0001$ \\
\hline & \multirow[t]{6}{*}{ PW } & \multirow[t]{6}{*}{0.8849} & \multirow[t]{6}{*}{1.96} & \multirow[t]{6}{*}{0.013} & \multirow[t]{6}{*}{0.67} & Model & 10 & 0.0692 & 0.0069 & 39.99 & $<.0001$ \\
\hline & & & & & & Error & 52 & 0.0090 & 0.0002 & & \\
\hline & & & & & & Corrected total & 62 & 0.0782 & & & \\
\hline & & & & & & $\mathrm{LD}$ & 6 & 0.0679 & 0.0113 & 65.34 & $<.0001$ \\
\hline & & & & & & MAF & 2 & 0.0009 & 0.0005 & 2.61 & 0.0834 \\
\hline & & & & & & Method & 2 & 0.0005 & 0.0002 & 1.31 & 0.2792 \\
\hline \multirow{27}{*}{$\begin{array}{l}\text { Main effects + first- } \\
\text { order interactions }\end{array}$} & \multirow[t]{9}{*}{ FL } & \multirow[t]{9}{*}{0.9742} & \multirow[t]{9}{*}{1.17} & 0.009 & 0.74 & Model & 38 & 0.0677 & 0.0018 & 23.82 & $<.0001$ \\
\hline & & & & & & Error & 24 & 0.0018 & 0.0001 & & \\
\hline & & & & & & Corrected total & 62 & 0.0695 & & & \\
\hline & & & & & & LD & 6 & 0.0127 & 0.0021 & 28.25 & $<.0001$ \\
\hline & & & & & & MAF & 2 & 0.0068 & 0.0034 & 45.44 & $<.0001$ \\
\hline & & & & & & Method & 2 & 0.0198 & 0.0099 & 132.28 & $<.0001$ \\
\hline & & & & & & $\mathrm{LD} \times$ method & 12 & 0.0126 & 0.0010 & 14.01 & $<.0001$ \\
\hline & & & & & & $\mathrm{LD} \times \mathrm{MAF}$ & 12 & 0.0139 & 0.0012 & 15.43 & $<.0001$ \\
\hline & & & & & & MAF $\times$ method & 4 & 0.0020 & 0.0005 & 6.74 & 0.0009 \\
\hline & NI & 0.9938 & 1.34 & 0.007 & 0.56 & Model & 38 & 0.2129 & 0.0056 & 100.89 & $<.0001$ \\
\hline & & & & & & Error & 24 & 0.0013 & 0.0001 & & \\
\hline & & & & & & Corrected total & 62 & 0.2142 & & & \\
\hline & & & & & & LD & 6 & 0.1780 & 0.0297 & 534.32 & $<.0001$ \\
\hline & & & & & & MAF & 2 & 0.0055 & 0.0028 & 49.62 & $<.0001$ \\
\hline & & & & & & Method & 2 & 0.0179 & 0.0090 & 161.3 & $<.0001$ \\
\hline & & & & & & LD $\times$ method & 12 & 0.0087 & 0.0007 & 13.04 & $<.0001$ \\
\hline & & & & & & $\mathrm{LD} \times \mathrm{MAF}$ & 12 & 0.0024 & 0.0002 & 3.55 & 0.004 \\
\hline & & & & & & MAF $\times$ method & 4 & 0.0004 & 0.0001 & 1.72 & 0.178 \\
\hline & PW & 0.9998 & 0.13 & 0.001 & 0.67 & Model & 38 & 0.0782 & 0.0021 & 2610.91 & $<.0001$ \\
\hline & & & & & & Error & 24 & 0.0000 & 0.0000 & & \\
\hline & & & & & & Corrected total & 62 & 0.0782 & & & \\
\hline & & & & & & LD & 6 & 0.0679 & 0.0113 & $14,349.8$ & $<.0001$ \\
\hline & & & & & & MAF & 2 & 0.0009 & 0.0005 & 572.6 & $<.0001$ \\
\hline & & & & & & Method & 2 & 0.0005 & 0.0002 & 287.19 & $<.0001$ \\
\hline & & & & & & LD $\times$ method & 12 & 0.0001 & 0.0000 & 13.55 & $<.0001$ \\
\hline & & & & & & $\mathrm{LD} \times \mathrm{MAF}$ & 12 & 0.0088 & 0.0007 & 935.42 & $<.0001$ \\
\hline & & & & & & MAF $\times$ method & 4 & 0.0000 & 0.0000 & 2.13 & 0.1078 \\
\hline
\end{tabular}

$R^{2}$ coefficient of determination, $C V$ coefficient of variation, $R M S E$ root mean square error, Mean intercept value of the transformed accuracy (Z), $F L$ days to flowering, $N I$ nitrogen balance index, $P W 100$ panicle weight, $L D$ linkage disequilibrium with 7 levels (LD $\leq 0.25$, LD $\leq 0.36$, $\mathrm{LD} \leq 0.49, \mathrm{LD} \leq 0.64, \mathrm{LD} \leq 0.81, \mathrm{LD} \leq 0.98, \mathrm{LD} \leq 1, M A F$ minor allele frequency with 3 levels (MAF $\geq 5 \%, \mathrm{MAF} \geq 10 \%, \mathrm{MAF} \geq 20 \%$ ), Method BayesB, GBLUP, RKHS 


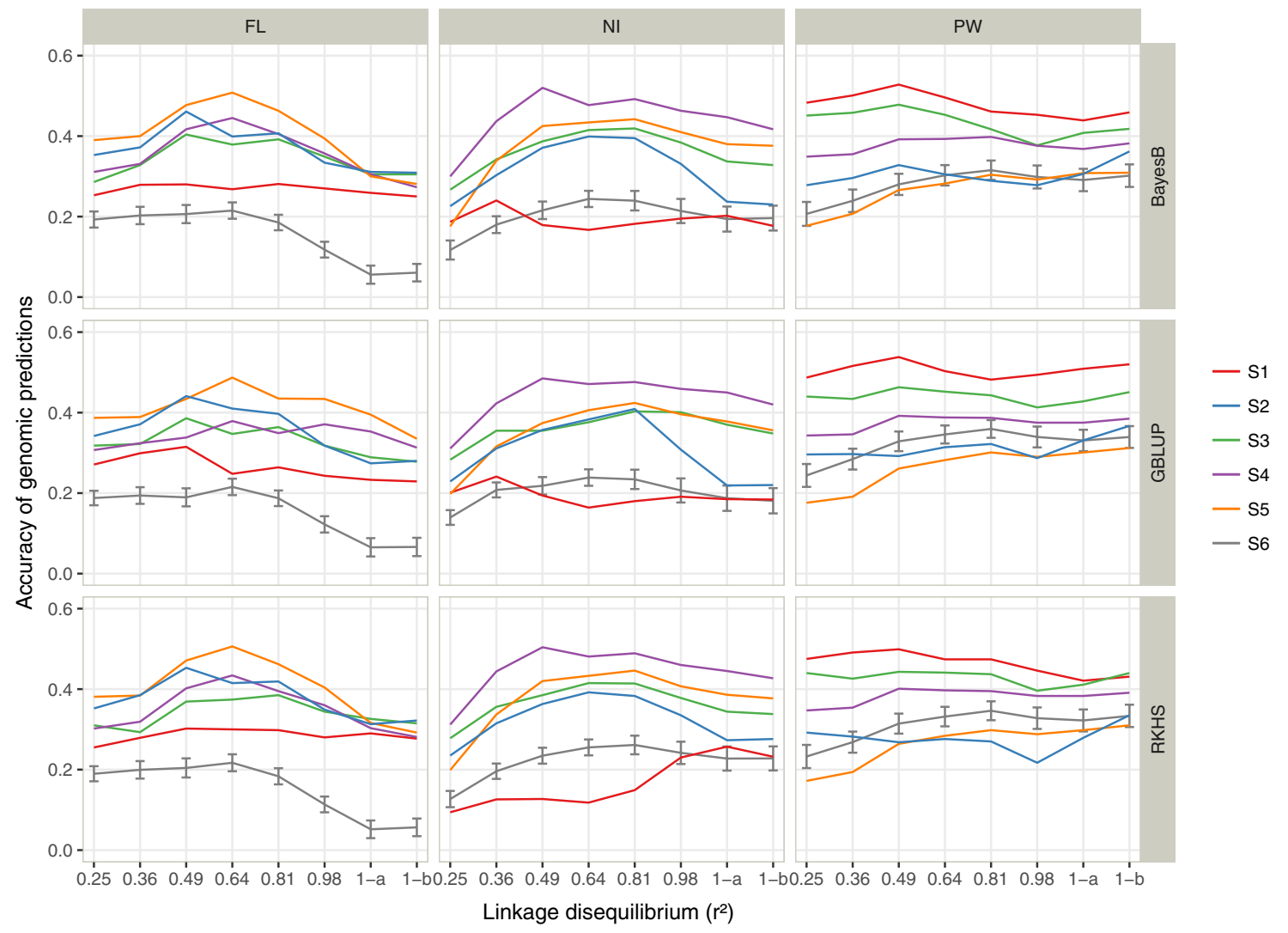

Fig. 5 Accuracy of genomic prediction of progeny phenotype for days to flowering (FL), nitrogen balance index (NI) and 100 panicle weight $(\mathrm{PW})$, obtained with three statistical methods, BayesB, GBLUP and RKHS. The six scenarios are described in Table 3.

to both the size and the degree of relatedness of the training set with the progeny set. Consequently, for each trait, the mean PAA obtained with the medium size training sets S3 (0.34, 0.36 and 0.43 , for FL, NI and PW, respectively) was not much below the one obtained with the largest training set S4 (0.35, 0.44 and 0.40, for FL, NI and PW, respectively). The presence of the parental line in the training set improved the PPA for NI and PW, as illustrated by the lower mean PPA of NI and PW in S2 (0.31), compared to the mean PPA of $\mathrm{NI}$ and PW in S3 (0.40), and the mean PPA of NI and PW in S5 (0.32), compared to S4 (0.42). This was not the case for FL (mean PPA of 0.37 in S2 and 0.34 in S3; mean PPA of 0.35 in S4 and 0.41 in S5), probably because the three prediction models we used could not capture the transgressive distribution of this trait observed in the progeny of the same crosses. Likewise, when the size of the training set was too small, like in $\mathrm{S} 1$ (training set $=31$ parental lines; mean PPA $=0.31$, good relatedness was not sufficient to obtain a similar PPA to that obtained with a larger training set.

To further explore the effect of relatedness between the training and the candidate set, we used the CDmean method (Rincent et al. 2012) to select the accessions to be included
For scenario S6 that includes random sampling, the average and the 95\% confidence interval are shown. 1-a and 1-b, represent incidence matrices with no selection on $r^{2}$, but filtered with MAF $>5 \%$ and MAF $>2.5 \%$, respectively

in the training set. Three training sets $(n=31, n=58$ and $n=89$, equivalent in size to scenarios $\mathrm{S} 1, \mathrm{~S} 2$ and $\mathrm{S} 3$, respectively) were selected using the CDmean method, and used to predict progeny. The results (Supplementary Fig. 5) showed almost no gain in accuracy for FL and PW with the CDmean method, and an almost systematic gain in accuracy of about 0.1 for NI.

The magnitude of variation in PPA in relation with LD was much narrower. The highest mean PPA (0.36) was achieved with LD thresholds of $r^{2} \leq 0.49$ to $r^{2} \leq 0.81$, when interactions with other factors were left aside. The PPA decreased smoothly with both lower and higher LD thresholds, and reached 0.28 for $r^{2} \leq 0.25$, and 0.31 for $r^{2} \leq 1$. The inclusion of additional markers under $r^{2} \leq 1$, by lowering the MAF threshold to $2.5 \%$, neither deteriorated nor improved the PPA (Supplementary Table 6).

As expected, the accuracy of progeny prediction within two individual crosses showed much larger variation $(-0.310$ to 0.731$)$ depending on the trait, the scenario and the size of the incidence matrix (Supplementary Figs. 6 and 7). Given the small number of progenies for each of the two crosses used for this analysis (20 and 9), drawing 
Table 6 ANOVA of factors influencing the transformed accuracy $(Z)$ of 126 progeny prediction experiments for three phenotypic traits

\begin{tabular}{|c|c|c|c|c|c|c|c|c|c|c|c|}
\hline$\overline{\text { Model }}$ & Trait & $R^{2}$ & $\mathrm{CV}$ & RMSE & Mean & Source & $d f$ & SS & MS & $F$ value & $\operatorname{Prob} F$ \\
\hline \multirow[t]{18}{*}{ Main effects only } & \multirow[t]{6}{*}{ FL } & \multirow[t]{6}{*}{0.855} & \multirow[t]{6}{*}{14.64} & \multirow[t]{6}{*}{0.036} & \multirow[t]{6}{*}{0.25} & Model & 13 & 0.862 & 0.066 & 50.77 & $<.0001$ \\
\hline & & & & & & Error & 112 & 0.146 & 0.001 & & \\
\hline & & & & & & Corrected total & 125 & 1.008 & & & \\
\hline & & & & & & LD & 6 & 0.188 & 0.031 & 24 & $<.0001$ \\
\hline & & & & & & Method & 2 & 0.003 & 0.002 & 1.22 & 0.2999 \\
\hline & & & & & & Scenario & 5 & 0.671 & 0.134 & 102.72 & $<.0001$ \\
\hline & \multirow[t]{6}{*}{ NI } & \multirow[t]{6}{*}{0.848} & \multirow[t]{6}{*}{12.48} & \multirow[t]{6}{*}{0.045} & \multirow[t]{6}{*}{0.36} & Model & 13 & 1.277 & 0.098 & 48.19 & $<.0001$ \\
\hline & & & & & & Error & 112 & 0.228 & 0.002 & & \\
\hline & & & & & & Corrected total & 125 & 1.505 & & & \\
\hline & & & & & & LD & 6 & 0.237 & 0.040 & 19.41 & $<.0001$ \\
\hline & & & & & & Method & 2 & 0.000 & 0.000 & 0.03 & 0.968 \\
\hline & & & & & & Scenario & 5 & 1.039 & 0.208 & 102 & $<.0001$ \\
\hline & \multirow[t]{6}{*}{ PW } & \multirow[t]{6}{*}{0.876} & \multirow[t]{6}{*}{12.17} & \multirow[t]{6}{*}{0.047} & \multirow[t]{6}{*}{0.39} & Model & 13 & 1.791 & 0.138 & 61.11 & $<.0001$ \\
\hline & & & & & & Error & 112 & 0.252 & 0.002 & & \\
\hline & & & & & & Corrected total & 125 & 2.043 & & & \\
\hline & & & & & & LD & 6 & 0.035 & 0.006 & 2.6 & 0.0213 \\
\hline & & & & & & Method & 2 & 0.003 & 0.001 & 0.6 & 0.5497 \\
\hline & & & & & & Scenario & 5 & 1.753 & 0.351 & 155.53 & $<.0001$ \\
\hline \multirow{27}{*}{$\begin{array}{l}\text { Main effects + first- } \\
\text { order interactions }\end{array}$} & \multirow[t]{9}{*}{ FL } & \multirow[t]{9}{*}{0.978} & \multirow[t]{9}{*}{7.79} & 0.019 & 0.25 & Model & 65 & 0.986 & 0.015 & 40.99 & $<.0001$ \\
\hline & & & & & & Error & 60 & 0.022 & 0.000 & & \\
\hline & & & & & & Corrected total & 125 & 1.008 & & & \\
\hline & & & & & & LD & 6 & 0.188 & 0.031 & 84.68 & $<.0001$ \\
\hline & & & & & & Method & 2 & 0.003 & 0.002 & 4.3 & 0.018 \\
\hline & & & & & & Scenario & 5 & 0.671 & 0.134 & 362.49 & $<.0001$ \\
\hline & & & & & & $\mathrm{LD} \times$ method & 12 & 0.014 & 0.001 & 3.06 & 0.002 \\
\hline & & & & & & $\mathrm{LD} \times$ scenario & 30 & 0.102 & 0.003 & 9.15 & $<.0001$ \\
\hline & & & & & & Method $\times$ scenario & 10 & 0.009 & 0.001 & 2.4 & 0.0181 \\
\hline & $\mathrm{NI}$ & 0.980 & 6.18 & 0.022 & 0.36 & Model & 65 & 1.475 & 0.023 & 45.45 & $<.0001$ \\
\hline & & & & & & Error & 60 & 0.030 & 0.000 & & \\
\hline & & & & & & Corrected total & 125 & 1.505 & & & \\
\hline & & & & & & LD & 6 & 0.237 & 0.040 & 79.21 & $<.0001$ \\
\hline & & & & & & Method & 2 & 0.000 & 0.000 & 0.13 & 0.8761 \\
\hline & & & & & & Scenario & 5 & 1.039 & 0.208 & 416.31 & $<.0001$ \\
\hline & & & & & & $\mathrm{LD} \times$ method & 12 & 0.006 & 0.001 & 1.01 & 0.4507 \\
\hline & & & & & & $\mathrm{LD} \times$ scenario & 30 & 0.173 & 0.006 & 11.57 & $<.0001$ \\
\hline & & & & & & Method $\times$ scenario & 10 & 0.019 & 0.002 & 3.78 & 0.0006 \\
\hline & PW & 0.972 & 7.93 & 0.031 & 0.39 & Model & 65 & 1.986 & 0.031 & 31.97 & $<.0001$ \\
\hline & & & & & & Error & 60 & 0.057 & 0.001 & & \\
\hline & & & & & & Corrected total & 125 & 2.043 & & & \\
\hline & & & & & & LD & 6 & 0.035 & 0.006 & 6.14 & $<.0001$ \\
\hline & & & & & & Method & 2 & 0.003 & 0.001 & 1.42 & 0.2499 \\
\hline & & & & & & Scenario & 5 & 1.753 & 0.351 & 366.83 & $<.0001$ \\
\hline & & & & & & $\mathrm{LD} \times$ method & 12 & 0.012 & 0.001 & 1.04 & 0.4256 \\
\hline & & & & & & $\mathrm{LD} \times$ scenario & 30 & 0.120 & 0.004 & 4.18 & $<.0001$ \\
\hline & & & & & & Method $\times$ scenario & 10 & 0.063 & 0.006 & 6.62 & $<.0001$ \\
\hline
\end{tabular}

$R^{2}$ coefficient of determination, $C V$ coefficient of variation, $R M S E$ root mean square error, Mean intercept value of the transformed accuracy (Z), $F L$ days to flowering, $N I$ nitrogen balance index, $P W 100$ panicle weight, $L D$ linkage disequilibrium with 7 levels (LD $\leq 0.25$, LD $\leq 0.36$, $\mathrm{LD} \leq 0.49, \mathrm{LD} \leq 0.64, \mathrm{LD} \leq 0.81, \mathrm{LD} \leq 0.98, \mathrm{LD} \leq 1), M A F$ minor allele frequency with 3 levels (MAF $\geq 5 \%, \mathrm{MAF} \geq 10 \%, \mathrm{MAF} \geq 20 \%$, Method BayesB, GBLUP, RKHS 
any conclusion regarding the effect of one individual factor (scenario, trait, LD) would be risky. However, the fact that accuracy above 0.7 was obtained in some conditions strongly suggests the feasibility of intra-cross progeny prediction using a diversity panel.

\section{Discussion}

The main objectives of this work were to assess the performance of genomic prediction among the progeny of biparental crosses, using a reference panel to train the model in rice, and to investigate the effect of the size of the reference panel and of the degree of relatedness with the progeny population on the accuracy of predictions, as well as the effect of $\mathrm{LD}$ and the training model. To set a base line for prediction accuracy and to reduce the number of possible options to be tested regarding $L D$ and other characteristics of the incidence matrix, we started our study by evaluating the accuracy of genomic prediction within the reference population using a cross validation approach.

\section{Accuracy of genomic prediction in the reference population}

The average genomic prediction accuracies within the reference population ranged from 0.51 for NI to 0.63 for FL, in line with their degree of broad sense heritability. The highest accuracies were 0.65 for FL, 0.57 for NI, and 0.62 for PW. Beyond the high heritability, the rather narrow genetic diversity of our RP assembling temperate and tropical japonica adapted to the irrigated lowland ecosystem of Europe, has probably contributed to the relatively high accuracy of genomic prediction for complex traits such as NI and PW.

The accuracy of genomic prediction was affected in a complex way by interactions between LD, MAF and phenotypic traits, but did not question the well-established rule of balance between the number and the distribution of markers along the chromosome, and the LD within the population (Jannink et al. 2010). However, the GBS genotyping method resulted in heterogeneous marker distribution, with distances between adjacent marker varying from one base to more than one Mb. The pruning of SNP markers based on LD information enabled us to improve accuracy with non-redundant SNP matrices. Our interpretation of the increase in prediction accuracy when marker redundancy was reduced is that: (1) the higher the number of redundant markers, the smaller the contribution of individual markers in the prediction model, including those tightly linked to the QTL or the most determining one for the calculation of genomic distance between individuals; (2) prediction models based on a high number of redundant markers are less accurate, as they capture numerous false genotype-phenotype relationships or build less discriminant genomic distances.

The trend towards an increase in prediction accuracy with a reduction in marker redundancy was best captured with GBLUP, raising the question of its origin, model formulation per se or method of implementation. Using the BGLR package (Pérez and de los Campos 2014) to fit GBLUP in a Bayesian framework with MCMC sampling (GBLUP_B), we explored the hypothesis of method of implementation. Our results revealed (Supplementary Fig. 8) a difference between the two methods with a plateau of accuracy for GBLUP_B for matrices with medium to high marker density, suggesting that the implementation of the GBLUP method was responsible of the decrease of accuracy rather than the method itself. This difference is probably related to a better convergence of the MCMC algorithm compared to the EM algorithm.

In the present study, we used a simple procedure based on pairwise LD to eliminate the most redundant markers. Other procedures have been developed: selection of tag SNPs based on LD, diversity or hot spots of recombination (Carlson et al. 2004; Zhang et al. 2004; Halperin et al. 2005), measuring the contribution of each marker with a statistic called 'degree of tagging', that includes both pairwise LD and base-pair distance (Speed et al. 2012; Ramstein et al. 2016). The practical lesson that can be drawn from our procedure is that the accuracy of prediction can be significantly improved by not including markers that constitute the largest high redundancy clusters.

Compared to $\mathrm{LD}$, the MAF and the prediction method had much more limited effects on prediction accuracy, although the effects were significant. The rather small MAF effect suggests that low MAF mainly results from random genetic drift (Edriss et al. 2012) and/or that markers closely linked with genes which affect our target traits, have not yet reached a high level of fixation within our population. Regarding the method effect, the lower performances of GBLUP for predicting FL and NI suggests the existence of QTLs with a rather large effect that could be better captured using methods based on marker effect than using information on genomic relationships.

\section{Accuracy of genomic prediction of progeny performances}

Our genomic prediction experiments on the line value of $\mathrm{F}_{5}-\mathrm{F}_{7}$ progenies of biparental crosses, each involving two accessions belonging to the reference population, mimicked a rice breeding scheme in which the breeding cycle is shortened by rapid generation advancement (RGA) of the early generations, and where the phenotypic evaluation starts with the advanced $\mathrm{F}_{5}$ or $\mathrm{F}_{6}$ generation. RGA consists in the fixation of $F_{2}$ progenies through 2-3 generations of 
single seed descent per year in the greenhouse, until $\mathrm{F}_{5}$ or $F_{6}$. However, our experiments diverged from this scheme by the very pronounced imbalance in the number of progeny per cross, which varied from 1 to 20 .

The accuracy of our progeny predictions among the 97 advanced lines of PP derived from 36 biparental crosses, involving 31 accessions of RP, varied greatly for each trait, depending on the composition of the training set and the LD. However, for each trait, the highest degree of accuracy achieved was only slightly below the highest accuracy achieved in the cross validation experiments in the RP: 0.51 versus 0.65 for FL, 0.52 versus 0.57 for NI and 0.54 versus 0.62 for PW. Similar results have been obtained in sugar beet (Hofheinz et al. 2012), in barley (Sallam et al. 2015), in wheat (Michel et al. 2016) and in strawberry (Gezan et al. 2017). What is more, in our case, rather high accuracies (up to 0.7 ) were obtained in progeny prediction among the full-sib lines of individual crosses. However, the number of progeny per cross and the number crosses analyzed were too small to draw general conclusions.

Population parameters that affect the accuracy of progeny prediction include differences in LD and allele frequency between RP and PP, as well as the parental contributions to PP and the genetic distance, or number of generations, between the two populations (Daetwyler et al. 2010; Lorenz et al. 2012). Recombination in breeding populations reduces LD between markers and QTLs over time, while selection increases LD (Pfaffelhuber et al. 2008). In our case, only one cycle of recombination separated RP and PP and the two populations did not differ much in either long distance LD or short distance LD, i.e., LD between markers and QTLs (Fig. 2). This is not particularly surprising given the composition of PP, involving a large number of biparental crosses. Moreover, the average LD $\left(r^{2}=0.2\right.$ at 850 and $1100 \mathrm{~kb}$ distance in RP and PP, respectively) was much higher than that reported in the literature for the japonica group (Courtois et al. 2013), suggesting narrower genetic diversity of the RP compared to the whole japonica group. Regarding the genetic distance between RP and PP, and the contributions of individual parental lines to the final composition of PP, marked unbalance was observed, to the advantage of the temperate japonica subgroup. Indeed, while the tropical japonica subgroup represented $24 \%$ of the accessions of RP, only $8 \%$ of PP lines clustered with the tropical japonica subgroup. Likewise, at the level of individual crosses, voluntary or involuntary selection of progenies skewed their distribution toward the temperate japonica genetic background. Indeed, while $38 \%$ of the 36 biparental crosses involved a tropical japonica accession of RP and produced more than $50 \%$ of the progeny lines of PP, only $15 \%$ of these progeny clustered with the tropical japonica subgroup. These unbalances raise the question of how to choose the individuals that make up the training set to maximize the accuracy of progeny predictions.

\section{Selection of the training set to optimize accuracy of progeny prediction}

Several studies have shown that the accuracy of genomic predictions is highly influenced by the degree of relatedness between TP and CP (Pszczola et al. 2012; Rincent et al. 2012; Hayes et al. 2009b). As discussed above, in our study, there was marked variation in the degree of relatedness between the individuals of the two populations. This large variation raised the question of the choice of the RP individuals to be included in the training set to maximize the accuracy of progeny predictions. The results of the six compositions of the training set scenarios we tested confirmed the complementary effects of relatedness between the training set and the PP, and the size of the training set. The lower mean PPA observed under scenario S1, compared to scenarios S3 and S4 shows that, in addition to relatedness between the training set and PP, the size of the training set also matters, and even distant accessions can positively contribute to prediction accuracy. The results of scenario S2 demonstrate that high APA can be achieved without the presence of the parental lines in the training set provided it is composed of individuals closely related to the parental lines. The highest APA observed under scenario S4 suggests there is still room for optimization of the size and the composition of the training set. For instance, by weighting the contribution of each parental line to the composition of the pools of the most closely and most distantly related individuals in the $\mathrm{RP}$, based on their actual contribution (ratio of the number of progeny to the total number of individuals in the PP) to the composition of the PP. The almost equal APA observed in S3 and S4 suggests that beyond a certain size threshold of the training set composed of accessions closely related to the PP, the inclusion of less closely related individuals does not improve prediction accuracy. These findings are in agreement with those of Pszczola et al. (2012), who showed that the relatedness between the reference individuals and between the candidates and the reference individuals has a strong effect on accuracy. Given the above-mentioned effects of selection on PP, one could expect better prediction accuracy with optimization methods that directly use information on relatedness between the individuals in the training set and the individuals in the PP, such as CDmean (Rincent et al. 2012). Comparison of accuracy obtained under scenarios S1, S2 and S3, with the accuracy obtained with the training set selected using the CDmean method only partially confirmed this expectation. This is probably due to the fact that our scenario for optimization of the training set was also based on relatedness between the training set and the parental lines of the PP. 
All our training set optimization experiments targeted genomic prediction among the progenies of 36 biparental crosses. In general, pedigree breeding programs target the progeny of individual biparental crosses. Most of the individual biparental populations in our data set were too small for such experiments. However, prediction accuracies of above 0.5 were observed for NI and WP under some LD thresholds and in some scenarios, even though the training sets were not specifically optimized for these populations. This encouraging result merits confirmation using a more appropriate data set.

When predicting GEBVs on progeny, the optimal size of the training set depends on the degree of relatedness (number of generations between the training set and the progeny set), the effective size of population $\mathrm{Ne}$, the length of the genetic map, and the architecture of the target trait (Jannink et al. 2010). Generally speaking, an increase in the size of the TP improves prediction accuracy, but in addition to size, the genetic structure of the TP and the relationship between this structure and the distribution of the target trait, also matter. For instance, Technow et al. (2013) observed a 10\% increase in prediction accuracy, when they combined data from two heterotic groups of corn (flint and dent) to predict resistance to leaf blight in one of the groups. Conversely, Lorenz et al. (2012) observed no significant improvement in the prediction of resistance to fusarium head blight and its associated resistance to mycotoxins, when they increased the size of the TP by combining different barley breeding populations. In the present study, the highest average accuracies were achieved with the largest training set for PW and NI traits that have complex genetic architecture. Prediction accuracy was less responsive to the size of the training set for the FL trait, of oligo-genic determinism (Hori et al. 2016).

\section{Practical implications for rice breeding programs}

Pedigree breeding within the progenies of biparental crosses extracted from a working collection or reference population is the most common scheme for the improvement of complex traits in rice, as in many other autogamous crops (Bernardo 2014). We found that, using phenotypic and genotypic data from the RP to train the prediction model made it possible to predict performances among the first generation of advanced $\left(\mathrm{F}_{5}-\mathrm{F}_{7}\right)$ progeny of a large set of biparental crosses. Accuracies of over 0.5 were obtained, even for complex traits such as NI and PW, when the parameters that affect the accuracy were optimized. Thus, breeders can use this prediction approach in the framework of a pedigree breeding scheme associated with RGA of early generations (in off-season nurseries or controlled environments), a practice aimed at reducing the length of the breeding cycle and hence accelerating genetic gain per unit of time (O'Connor et al. 2013).
However, specific optimization of the training set might be needed to obtain the best possible prediction accuracy for the progeny of each cross. The scheme can also be applied in breeding schemes that use the haplo-diploidization method for the rapid generation of homozygous lines from biparental crosses, at least in the japonica genetic group for which a high-throughput haplo-diploidization method is available (Alemanno and Guiderdoni 1994). As the advanced line selected in this way will then go through 2-3 cycles of phenotypic evaluation, the data collected will provide an opportunity to further refine the training model (Heffner et al. 2010).

We also found that (1) an average marker density above one per $22 \mathrm{~kb}$ (8324 SNPs) did not improve the accuracy of prediction in either cross validation within the RP or in progeny prediction and (2) relatively high accuracy could be achieved using only a rather small share of the RP, most related to PP, as the training set. Given the very uneven distribution of marker density along the chromosomes in our RP and PP, one would expect similar levels of prediction accuracy with a much smaller number of markers chosen based on LD distribution along the chromosomes, as already predicted in simulation studies (Habier et al. 2009; Lillehammer and Meuwissen 2013; Grattapaglia 2014). These findings attest to the feasibility of using the genomic selection approach in breeding programs with rather limited resources. The most efficient and affordable option would be rather dense genotyping of the RP accessions and much looser (a few hundred), but evenly distributed, genotyping of PP that can be densified through imputation, a method widely practiced in animal breeding (Marchini and Howie 2010).

Author contributions NA and GV conceived the study. CC extracted the DNA and prepared the library for GBS. GO, JR, LR, CB, CB, AV, FD, MBH implemented the field trials. $\mathrm{MBH}, \mathrm{TVC}, \mathrm{JB}, \mathrm{LJ}, \mathrm{NA}$ analyzed the data. MBH and NA wrote the manuscript. TVC and JB revised the manuscript.

Acknowledgements This work was funded by Agropolis Foundation (http://www.agropolis-fondation.fr/) and Cariplo Foundation (http:// www.fondazionecariplo.it/), Grant no. 1201-006.

\section{Compliance with ethical standards}

Conflict of interest The authors declare that they have no conflict of interest.

Open Access This article is distributed under the terms of the Creative Commons Attribution 4.0 International License (http://creativecommons.org/licenses/by/4.0/), which permits unrestricted use, distribution, and reproduction in any medium, provided you give appropriate credit to the original author(s) and the source, provide a link to the Creative Commons license, and indicate if changes were made. 


\section{References}

Akdemir D, Sanchez JI, Jannink JL (2015) Optimization of genomic selection training populations with a genetic algorithm. Genet Select Evol 47:38. https://doi.org/10.1186/s12711-015-0116-6

Albrecht T, Wimmer V, Auinger HJ, Erbe M, Knaak C et al (2011) Genome-based prediction of testcross values in maize. Theor Appl Genet 123:339-350

Alemanno L, Guiderdoni E (1994) Increased doubled haploid plant regeneration from rice (Oryza sativa L.) anthers cultured on colchicine-supplemented media. Plant Cell Rep 13(8):432-436. https://doi.org/10.1007/BF00231961

Ashikari M (2017) Advances in molecular breeding techniques for rice. In Sasaki T (ed) Achieving sustainable cultivation of rice, vol. 1. Breeding for higher yield and quality. 280 p. www. bdspublishing.com

Atlin G, Cairns GE, Das B (2017) Rapid breeding and varietal replacement are critical to adaptation of cropping systems in the developing world to climate change. Glob Food Sec 12(2017):31-37. https://doi.org/doi.org/10.1016/j. gfs.2017.01.008

Bernardo R (2014) Essentials of plant breeding. Stemma Press, p 252. http://stemmapress.com. ISBN 978-0-9720724-2-7

Beyene Y, Semagn K, Mugo S, Tarekegne A, Babu R et al (2015) Genetic gains in grain yield through genomic selection in eight biparental maize populations under drought stress. Crop Sci 55:154

Biscarini F, Cozzi P, Casella L, Riccardi P, Vattari A, Orasen G et al (2016) Genome-wide association study for traits related to plant and grain morphology, and root architecture in temperate rice accessions. PLoS One 11(5):e0155425. https://doi.org/10.1371/ journal.pone.0155425

Burstin J, Salloignon P, Martinello M, Magnin-Robert J-B, Siol M, Jacquin $\mathrm{F}$ et al (2015) Genetic diversity and trait genomic prediction in a pea diversity panel. BMC Genom 16:105. https:// doi.org/10.1186/s12864-015-1266-1

Carlson CS, Eberle MA, Rieder MJ, Yi Q, Kruglyak L, Nickerson DA (2004) Selecting a maximally informative set of singlenucleotide polymorphisms for association analyses using linkage disequilibrium. Am J Hum Genet 74(1):106-120

Courtois B, Audebert A, Dardou A, Roques S, Ghneim Herrera T, Droc G, Frouin J, Gozé E, Kilian A, Ahmadi N, Dingkhun M (2013) Genome-wide association mapping for root depth in a japonica rice panel. PLoS One 8(11):e78037. https://doi. org/10.1371/journal.pone.0078037

Daetwyler HD, Pong-Wong R, Villanueva B, Woolliams JA (2010) The impact of genetic architecture on genome-wide evaluation methods. Genetics 185:1021-1031. https://doi.org/10.1534/ genetics.110.116855

Desta ZA, Ortiz R (2014) Genomic selection: genome-wide prediction in plant improvement. Trends Plant Sci 19(9):292-601. https://doi.org/doi.org/10.1016/j.tplants.2014.05.006

Edriss V, Guldbrandtsen B, Lund MS, Su G (2012) Effect of marker-data editing on the accuracy of genomic prediction. J Anim Breed Genet 130(2013):128-135. https://doi. org/10.1111/j.1439-0388.2012.01015.x

Faivre-Rampant O, Bruschi G, Abbruscato P, Cavigiolo S, Picco AM, Borgo L et al (2011) Assessment of genetic diversity in Italian rice germplasm related to agronomic traits and blast resistance (Magnaporthe oryzae). Mol Breed 27:233-246

Gezan SA, Osorio LF, Verma S, Whitaker VM (2017) An experimental validation of genomic selection in octoploid strawberry. Hortic Res 4:16070. https://doi.org/10.1038/hortres.2016.70

Gianola D, van Kaam JBCHM (2008) Reproducing kernel Hilbert spaces regression methods for genomic assisted prediction of quantitative traits. Genetics 178(4):2289-2303
Glaubitz JC, Casstevens TM, Lu F, Harriman J, Elshire RJ, Sun Q, Buckler SE (2014) TASSEL-GBS: a high capacity genotyping by sequencing analysis pipeline. PLoS One 9(2):e90346. https:// doi.org/10.1371/journal.pone.0090346

Goulas Y, Cerovic ZG, Cartelat A, Moya I (2004) Dualex: a new instrument for field measurements of epidermal ultraviolet absorbance by chlorophyll fluorescence. Appl Opt 43(23):4488-4496

Grattapaglia D (2014) Breeding forest trees by genomic selection: current progress and the way forward. In: Tuberosa R, Graner A Frison E (eds) Genomics of plant genetic resources. Springer, The Netherlands, pp 651-682

Grenier C, Cao T-V, Ospina Y, Quintero C, Châtel MH, Tohme J, Courtois B, Ahmadi N (2015) Accuracy of genomic selection in a rice synthetic population developed for recurrent selection breeding. PLoS One 10(8):e0136594. https://doi.org/10.1371/ journal.pone.0136594

Guo Z, Tucker DM, Basten CJ, Gandhi H, Ersoz E, Guo B et al (2014) The impact of population structure on genomic prediction in stratified populations. Theor Appl Genet 127:749-762. https:// doi.org/10.1007/s00122-013-2255-x

Habier D, Fernando RL, Dekkers JMC (2009) genomic selection using low-density marker panels. Genet Soci Am. https://doi. org/10.1534/genetics.108.100289

Halperin E, Kimmel G, Shamir R (2005) Tag SNP selection in genotype data for maximizing SNP prediction accuracy. Bioinformatics 21(suppl 1):i195-i203

Hayes B, Bowman P, Chamberlain A, Goddard M (2009a) Invited review: genomic selection in dairy cattle: progress and challenges. J Dairy Sci 92(2):433-443. https://doi.org/10.3168/jds.2008-1646

Hayes BJ, Bowman P, Chamberlain A, Verbyla K, Goddard M (2009b) Accuracy of genomic breeding values in multi-breed dairy cattle populations. Genet Select Evol 41:51

Heffner EL, Lorenz AJ, Jannink J-L, Sorrells ME (2010) Plant breeding with genomic selection: gain per unit time and cost. Crop Sci 50:1681-1690. https://doi.org/doi.org/10.2135/ cropsci2009.11.0662

Heffner EL, Jannink JL, Sorrells ME (2011) Genomic selection accuracy using multifamily prediction models in a wheat breeding program. Plant Genome 4:65-75. https://doi.org/10.3835/ plantgenome.2010.12.0029

Heslot N, Yang H-P, Sorrells ME, Jannink J-L (2012) Genomic selection in plant breeding: a comparison of models. Crop Sci $52: 146-160$

Heslot N, Rutkoski J, Poland J, Jannink JL, Sorrells ME (2013) Impact of marker ascertainment bias on genomics election accuracy and estimates of genetic diversity. PLoS One 8:e74612. https://doi. org/10.1371/journal.pone.0074612

Hofheinz N, Borchardt D, Weissleder K, Frisch M (2012) Genome based prediction of test cross performance in two subsequent breeding cycles. Theor Appl Genet 125:1639-1645. https://doi. org/10.1007/s00122-012-1940-5

Holland JB, Nyquist WE, Cervantes-Martinez CT (2003) Estimating and interpreting heritability for plant breeding: an update. Plant Breed Rev 22:9-111

Hori K, Matsubara K, Yano M (2016) Genetic control of flowering time in rice: integration of Mendelian genetics and genomics. Theor Appl Genet 129:2241. https://doi.org/10.1007/s00122-016-2773-4

Howard R, Carriquiry AL, Beavis WD (2014) Parametric and nonparametric statistical methods for genomic selection of traits with additive and epistatic genetic architectures. Genes Genom Genet 4(6):1027-1046. https://doi.org/10.1534/g3.114.010298

Isidro J, Jannink J-L, Akdemir D, Poland J, Heslot N, Sorrells ME (2015) Training set optimization under population structure in genomic selection. Theor Appl Genet 128:145-158. https://doi. org/10.1007/s00122-014-2418-4 
Jacquin L, Cao T-V, Ahmadi N (2016) A unified and comprehensible view of parametric and kernel methods for genomic prediction with application to Rice. Front Genet 7:145. https://doi. org/10.3389/fgene.2016.00145

Jannink JL, Lorenz AJ, Iwata H (2010) Genomic selection in plant breeding: from theory to practice. Brief Funct Genom Proteom 9:166-177. https://doi.org/10.1093/bfgp/elq001

Jarquín D, Kocak K, Posadas L, Hyma K, Jedlicka J, Graef G et al (2014) Genotyping by sequencing for genomic prediction in a soybean breeding population. BMC Genom 15:740. https://doi. org/10.1186/1471-2164-15-740

Karatzoglou A, Smola A, Hornik K, Zeileis A (2004). Kernlab-an S4 package for Kernel methods in R. 1(9):20

Lillehammer M, Meuwissen THE (2013) Sonesson AK (2013), A lowmarker density implementation of genomic selection in aquaculture using within-family genomic breeding values. Genet Sel Evol 45(45):39. https://doi.org/10.1186/1297-9686-45-39 (Published online)

Lorenz AJ, Chao S, Asoro GF, Heffner LF, Hayashi T, Iwata H, Smith KP, Sorrells ME, Jannink J-L (2011) Genomic selection in plant breeding: knowledge and prospects. Adv Agron 110:77-123

Lorenz AJ, Smith KP, Jannink J-L (2012) Potential and optimization of genomic selection for Fusarium head blight resistance in sixrow barley. Crop Sci 52:1609-1621. https://doi.org/10.2135/ cropsci2011.09.0503

Lorenzana RE, Bernardo B (2009) Accuracy of genotypic value predictions for marker-based selection in biparental plant populations. Theor Appl Genet 120:151-161

Marchini J, Howie B (2010) Genotype imputation for genome-wide association studies. Nat Rev Genet 11:499-511

Meuwissen TH, Hayes BJ, Goddard ME (2001) Prediction of total genetic value using genome-wide dense marker maps. Genetics 157:1819-1829

Michel S, Ametz C, Gungor H, Epure D, Grausgruber H, Löschenberger F, Buerstmayr H (2016) Genomic selection across multiple breeding cycles in applied bread wheat breeding. Theor Appl Genet 129:1179-1189. https://doi.org/10.1007/ s00122-016-2694-2

O'Connor DJ, Wright GC, Dieters MJ, George DL, Hunter MN, Tatnell JR, Fleischfresser DB (2013) Development and application of speed breeding technologies in a commercial peanut breeding program. Peanut Sci 40(2):107-114. https://doi.org/10.3146/PS12-12

Onogi A, Ideta O, Inoshita Y, Ebana K, Yoshioka T, Yamasaki M et al (2014) Exploring the areas of applicability of whole-genome prediction methods for Asian rice (Oryza sativa L.). Theor Appl Genet 128:41-53. https://doi.org/10.1007/s00122-014-2411-y

Pérez P, de los Campos G (2014) Genome-wide regression and prediction with the BGLR statistical package. Genetics 198(2):483-495. https://doi.org/10.1534/genetics.114.164442

Perrier X, Jacquemoud-Collet JP (2006) Darwin software. Centre de Cooperation Internationale en Recherche Agronomique pour le Developpement, Montpellier, France. http://darwin.cirad.fr/

Pfaffelhuber P, Lehnert A, Stephan W (2008) Linkage disequilibrium under genetic hitchhiking in finite populations. Genetics 179:527537. https://doi.org/10.1534/genetics.107.081497

Poland J, Endelman J, Dawson J, Rutkoski J, Wu S, Manes Y et al (2012) Genomic selection in wheat breeding using genotypingby-sequencing. Plant Genome 5:103-113. https://doi.org/10.3835/ plantgenome2012.06.0006
Pszczola M, Strabel T, Mulder H, Calus M (2012) Reliability of direct genomic values for animals with different relationships within and to the reference population. J Dairy Sci 95:389-400

Ramstein GP, Evans J, Kaeppler SM, Mitchell RM, Kenneth P, Voge KP, Robin Buel CR, Casler MD (2016) Accuracy of genomic prediction in switchgrass (Panicum virgatum L.) improved by accounting for linkage disequilibrium. G3 6:1049-1062. https:// doi.org/10.1534/g3.115.024950/-/DC1

Rincent R, Laloë D, Nicolas S, Altmann T, Brunel D, Revilla P, Rodríguez VM, Moreno- Gonzalez J, Melchinger A, Bauer E, Schoen CC, Meyer N, Giauffret C, Bauland C, Jamin P, Laborde J, Monod H, Flament P, Charcosset A, Moreau L (2012) Maximizing the reliability of genomic selection by optimizing the calibration set of reference individuals: Comparison of methods in two diverse groups of maize inbreds (Zea mays L.). Genetics 192(2):715-728. https://doi.org/10.1534/genetics.112.141473

Rogers AR, Huff C (2009) Linkage disequilibrium between loci with unknown phase. Genetics 182:839-844. https://doi.org/10.1534/ genetics.108.093153

Sallam A, Endelman J, Jannink JL, Smith K (2015) Assessing genomic selection prediction accuracy in a dynamic barley breeding population. Plant Genome 8:1. https://doi.org/10.3835/ plantgenome2014.05.0020

Speed D, Hemani G, Johnson RM, Balding JD (2012) Improved heritability estimation from genome-wide SNPs. Am J Hum Genet 91(6):1011-1021

Spindel J, Begum H, Akdemir D, Virk P, Collard B, Redoña E et al (2015) Genomic selection and association mapping in rice (Oryza sativa): effect of trait genetic architecture, training population composition, marker number and statistical model on accuracy of rice genomic selection in elite, tropical rice breeding lines. PLoS Genet. 11:e1004982. https://doi.org/10.1371/journal. pgen.1004982

Technow F, Bürger A, Melchinger AE (2013) Genomic prediction of northern corn leaf blight resistance in maize with combined or separated training sets for heterotic groups. G3 3(2):197-203. https://doi.org/10.1534/g3.112.004630

Tremblay N, Wang Z, Cerovic ZG (2012) Sensing crop nitrogen status with fluorescence indicators. A review. Agron Sustain Dev 32(2):451-464

VanRaden PM (2008) Efficient methods to compute genomic predictions. J Dairy Sci 91(11):4414-4423

Wang X, Li L, Yang Z, Zheng X, Yu S, Xu C, Hu Z (2017) Predicting rice hybrid performance using univariate and multivariate GBLUP models based on North Carolina mating design II. Heredity 118:302-310

Zhang K, Qin ZS, Liu JS, Chen T, Waterman MS, Sun F (2004) Haplotype block partitioning and tag SNP selection using genotype data and their applications to association studies. Genome Res 14(5):908-916

Zhang X, Pérez-Rodríguez P, Burgueño J, Olsen M, Buckler E, Atlin G, Prasanna BM, Vargas M, San Vicente F, Crossa J (2017) Rapid cycling genomic selection in a multiparental tropical maize population. Genes Genomes Genet 7:2315. https://doi.org/10.1534/ g3.117.043141

Zhong S, Dekkers J, Fernando RL, Jannink JL (2009) Factors affecting accuracy from genomic selection in populations derived from multiple inbred lines: a barley case study. Genetics 182:355-364 